\title{
COUPLED STOKES-DARCY MODEL WITH BEAVERS-JOSEPH INTERFACE BOUNDARY CONDITION*
}

\author{
YANZHAO $\mathrm{CAO}^{\dagger}$, MAX GUNZBURGER ${ }^{\ddagger}$, FEI HUA ${ }^{\S}$, AND XIAOMING WANG
}

Dedicated to Andrew Majda on the occasion of his $60^{\text {th }}$ birthday

\begin{abstract}
We investigate the well-posedness of a coupled Stokes-Darcy model with BeaversJoseph interface boundary conditions. In the steady-state case, the well-posedness is established under the assumption of a small coefficient in the Beavers-Joseph interface boundary condition. In the time-dependent case, the well-posedness is established via an appropriate time discretization of the problem and a novel scaling of the system under an isotropic media assumption. Such coupled systems are crucial to the study of subsurface flow problems, in particular, flows in karst aquifers.
\end{abstract}

Key words. Stokes and Darcy system, fluid and porous media flow, Beavers-Joseph interface boundary condition, well-posedness, time discretization, finite element approximation.

AMS subject classifications. 35Q35, 65M60, 76D07, 76S05, 86A05.

\section{Introduction}

Groundwater systems are of great importance to our daily lives. In many states within the United States as well as in many other nations, groundwater is a major source of drinkable and industrial water. Groundwater systems are so tightly bonded with the lives of human beings that they are also very susceptible to contamination. Great concerns have grown about the sustainability of groundwater systems and their self-cleansing ability.

Among groundwater systems, karst aquifers are one important type. Such aquifers are mostly made up of a matrix, i.e., a porous medium, that holds the water. This is usually referred to as the first porosity. However, underground fissures, conduits, surface sinkholes, and springs play a major role in fluid transport in karst aquifers, even though they occupy a much smaller space relative to the more homogeneously porous matrix in which the first porosity dominates. Traditional models for studying groundwater such as dual porosity models oversimplify the intricate, heterogeneous system and can accurately handle fluid transport mechanisms only in the matrix. It is impractical to use them for studying complicated systems like karst aquifers. The important second and third porosities are ignored from these models despite the simple fact that they are the major underground highways for water. Now, scientists are beginning to shed light on using the Navier-Stokes equations to tackle the highly structured second and tertiary porosities which are prevalent in real-world karst aquifers.

Numerous previous studies have endeavored to study the interaction between the free flow in the second and tertiary porosity (say conduits) and the confined flow in the matrices. Most of them are divided into three major categories: using the domain

\footnotetext{
* Received: February 13, 2008; accepted (in revised version): July 29, 2008.

†Department of Mathematics and Statistics, Auburn University Auburn, AL 36830, (caoy@ scs.fsu.edu).

¥Department of Scientific Computing, Florida State University, Tallahassee FL 32306-4120, (gunzburg@fsu.edu).

$\S$ Department of Mathematics and School of Computational Science, Florida State University, Tallahassee FL 32306-4120, (fhua@math.fsu.edu).

『Department of Mathematics, Florida State University, Tallahassee FL 32306-4120, (wxm@ math.fsu.edu), corresponding author.
} 
decomposition method [1, 2, 3, 4], using the Lagrange multiplier approach [5,6], or the two-grid method [7]. To sum up, in free flow, the Navier-Stokes equations or their linearized version, the Stokes equations, are commonly used. In the matrix, one popular choice is to use Darcy's law. For the coupled Navier-Stokes-Darcy or the linearized Stokes-Darcy models, two boundary conditions are well-accepted: the continuity of the normal velocity across the interface which is a consequence of the conservation of mass and the balance of force normal to the interface (2.8). In the three-dimensional case, two more interface conditions are needed. Here, we adopt the classical empirical Beavers-Joseph interface boundary condition, which was proposed in the seminal work [8]. Roughly speaking, Beavers and Joseph proposed that the tangential component of the normal stress of the flow in the conduit at the interface is proportional to the jump of the tangential velocity across the interface (2.8).

Although there is abundant empirical evidence supporting the validity of the Beavers-Joseph interface boundary, we are not aware of any rigorous mathematical work based on this interface boundary condition. The main mathematical difficulty in adopting the Beavers-Joseph interface boundary condition seems to be the fact that this condition makes an indefinite leading order contribution to the total energy budget. Previous mathematical works on the coupled Stokes-Darcy system all used simplified interface boundary conditions such as the Beavers-Joseph-Saffman-Jones interface boundary condition [6] which basically neglects the contribution of the flow in the porous media to the interface boundary condition (2.9), or an even simpler interface boundary condition [3] (which is similar to a "free-slip" boundary condition) which is obtained by setting a coefficient to zero in the Beavers-Joseph interface boundary condition (2.8). All these simplified interface boundary conditions imply that the contribution of the interface boundary condition to the total energy budget is dissipative and hence analysis are possible. One of the main contributions of this paper is a novel scaling (which may be interpreted as pre-conditioning) for the coupled system so that the indefinite contribution from the interface term is controlled by the dissipation terms to the leading order (a Gårding type estimate). This essentially leads to the well-posedness of the system.

There exists substantial evidence supporting the usage of simplified interface boundary conditions. For instance, Saffman [9] and Jones [10] proposed the simplified interface boundary condition which bears their names based on consideration of very special cases and an ad hoc asymptotic analysis. Saffman considered one-dimensional flow under a uniform pressure gradient (which means no mass exchange between the conduit and the matrix) in uniform media (isotropic and homogeneous and hence constant hydraulic conductivity) and in the zero permeability limit. The simplified interface condition was mathematically validated by Jäger and Mikelic̀ [11] in the sense that the leading order interface boundary condition is the Beavers-Joseph-SaffmanJones boundary condition in the zero permeability limit under the similar assumptions as in Saffman's work plus the additional assumption that the flow is periodic in the horizontal direction (2D case). However, these assumptions may not necessarily hold in the sophisticated system such as a real-world karst aquifer. Fluid exchange between the conduit and the matrix, heterogenous and not necessarily small hydraulic permeability, and non-periodic boundary conditions are common in real-world problems and thus must be incorporated into the modeling to obtain useful results. We are not sure if the simplified Beavers-Joseph-Saffman-Jones interface boundary condition remains true in this kind of environment. Therefore it is natural to consider the coupled Stokes-Darcy system with the complete Beavers-Joseph interface boundary 
condition.

We also point out that all previous works emphasized the time-independent, steady state case. With real-world application in mind, especially the influence of precipitation which is reflected in the time dependent inflow-outflow boundary conditions in the conduit (see (2.7)), we mainly focus on time-dependent problems, although the time-independent case is also considered. It turns out that the time dependency is a blessing in our analysis since the dissipation terms are only able to control the leading order indefinite contribution from the Beavers-Joseph interface term.

The rest of the paper is organized as follows. We present the linear Stokes-Darcy model in their primitive variables as well as its weak formulation in section 2 with the full Beavers-Joseph interface boundary conditions. Section 3 is devoted to the stationary case. The well-posedness as well as a brief discussion of error estimates for finite element approximations are given in section 3 when the coefficient in the BeaversJoseph interface boundary condition is small enough. We tackle the time-dependent case in section 4 where a backward-Euler time discretization and a suitable scaling of the Darcy system are utilized to show the well-posedness. As a byproduct, we also derive a fully discrete scheme and show that it converges. However, a convergence rate is not given here. We consider convergence rates for finite element approximation in [12].

\section{The Stokes-Darcy system with the Beavers-Joseph interface condi- tion}

We begin with giving a full description of the problem we consider. figure 2.1

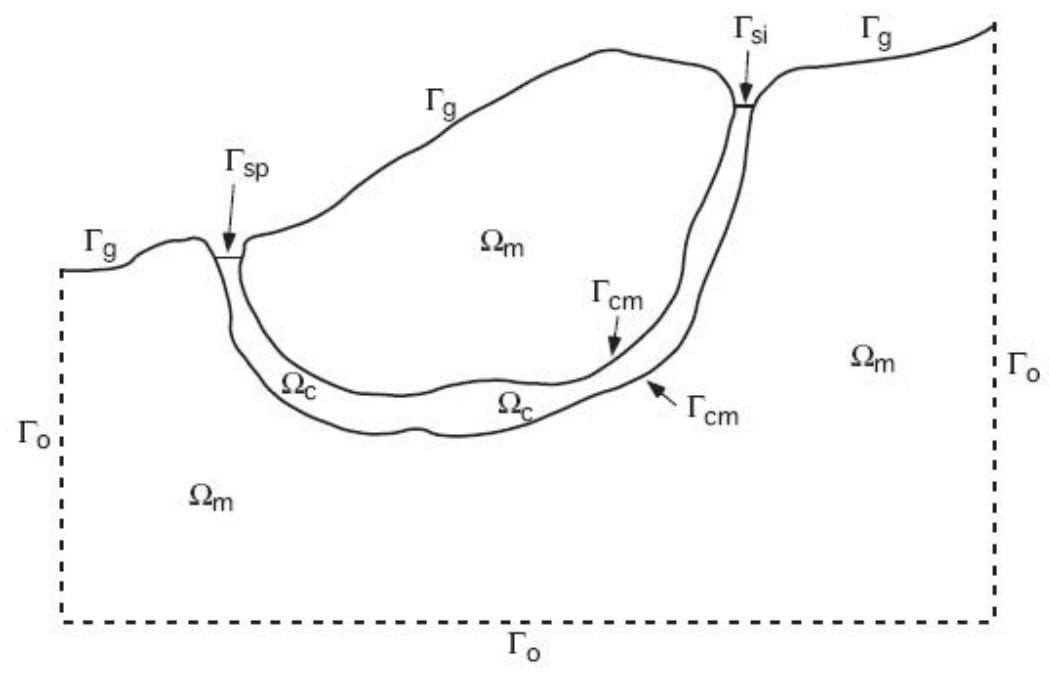

FIG. 2.1. Typical components of a karst aquifer.

depicts a simplified typical karst aquifer system. The free flow is confined in the underground conduit, denoted by $\Omega_{c}$, which connects a sinkhole to a spring. Surrounding the conduit are porous media such as soil, gravel, sand, etc. The porous media as a whole is regarded as the matrix that holds water. The region occupied by 
the matrix is denoted by $\Omega_{m}$. The flow in the matrix $\Omega_{m}$ is governed by

$$
\left.\begin{array}{rl}
S \frac{\partial \phi_{m}}{\partial t}+\nabla \cdot \mathbf{v}_{m} & =0 \\
\mathbf{v}_{m} & =-\mathbb{K} \nabla \phi_{m}
\end{array}\right\} \quad \text { in } \Omega_{m}
$$

which includes, in the first equation, the saturated flow model and, in the second equation, Darcy's law [13]. In (2.1), $S$ denotes the mass storativity coefficient, $\mathbb{K}(\mathbf{x})$ denotes the hydraulic conductivity tensor of the porous media, which is assumed to be symmetric and positive definite but could be location dependent (heterogeneous), and the unknown $\phi_{m}$ denotes the hydraulic (piezometric) head, defined as $\phi_{m}:=z+\frac{p_{m}}{\rho g}$, where $p_{m}$ represents the dynamic pressure, $z$ the height, $\rho$ the density, and $g$ the gravitational constant. Here the subscript $m$ emphasizes that these variables are for the matrix. We may omit this subscript where the context is clear. Combining the two equations in (2.1), we recover the heat equation for the hydraulic head:

$$
S \frac{\partial \phi_{m}}{\partial t}+\nabla \cdot\left(-\mathbb{K} \nabla \phi_{m}\right)=0 \quad \text { in } \Omega_{m}
$$

In the following, we will refer to (2.2) simply as the Darcy equation. We impose the following boundary conditions along the boundary of the matrix:

$$
\left\{\begin{aligned}
\phi_{m} & =f_{g} & & \text { on } \Gamma_{g} \\
\left(\mathbb{K} \nabla \phi_{m}\right) \cdot \mathbf{n} & =0 & & \text { on } \Gamma_{0},
\end{aligned}\right.
$$

the first of which naturally implies that the hydraulic head is specified to be $f_{g}$ at the ground surface and the second, by virtue of Darcy's law (the second equation in $(2.1)$ ), is the condition of no-flow across the boundary that is presumably a reasonable fictitious boundary condition useful for analysis and simulation purposes. We will consider the simple case of

$$
f_{g} \equiv 0
$$

in this manuscript. The general case can be handled by subtracting a background hydraulic head profile that fits the specified head at the ground surface. This will lead to a heat equation for the translated head with a source term which impose no difficulty in terms of analysis or computation.

In the conduit $\Omega_{c}$, the other domain of the problem, the Navier-Stokes equations govern the free flow:

$$
\left.\begin{array}{rl}
\frac{\partial \mathbf{v}_{c}}{\partial t}+\left(\mathbf{v}_{c} \cdot \nabla\right) \mathbf{v}_{c} & =\nabla \cdot\left(-p \mathbb{I}+2 \nu \mathbb{D}\left(\mathbf{v}_{c}\right)\right)-g \mathbf{k} \\
\nabla \cdot \mathbf{v}_{c} & =0
\end{array}\right\} \quad \text { in } \Omega_{c}
$$

where $\mathbf{v}_{c}$ denotes the flow velocity, $p$ the kinematic pressure, $\mathbb{D}(\mathbf{v})=\frac{1}{2}\left(\nabla \mathbf{v}+(\nabla \mathbf{v})^{T}\right)$ the deformation tensor, and $\mathbf{k}$ the unit vector in $z$ direction. Here the subscript $c$ emphasizes that these variables are for the conduit. We may omit this subscript where the context is clear. In this paper, we assume that the value of the Reynolds number is small so that we are able to replace the Navier-Stokes system by the linear Stokes system

$$
\left.\begin{array}{rl}
\frac{\partial \mathbf{v}_{c}}{\partial t} & =\nabla \cdot\left(-p \mathbb{I}+2 \nu \mathbb{D}\left(\mathbf{v}_{c}\right)\right)-g \mathbf{k}, \\
\nabla \cdot \mathbf{v}_{c} & =0
\end{array}\right\} \quad \text { in } \Omega_{c} .
$$


At the sinkhole and the spring, we use nonhomogeneous Dirichlet boundary conditions to specify inflow and outflow velocities:

$$
\left\{\begin{array}{llll}
\mathbf{v}_{c} \times \mathbf{n}=\mathbf{0} & \text { and } & \mathbf{v}_{c} \cdot \mathbf{n}=\gamma_{s i}(t) \eta_{s i}(\mathbf{x})=f_{s i} & \text { on } \Gamma_{s i} \\
\mathbf{v}_{c} \times \mathbf{n}=\mathbf{0} & \text { and } & \mathbf{v}_{c} \cdot \mathbf{n}=\gamma_{s p}(t) \eta_{s p}(\mathbf{x})=f_{s p} & \text { on } \Gamma_{s p}
\end{array}\right.
$$

where $\gamma, \eta$, and $f$ are given functions defined at the spring and the sinkhole, and $\mathbf{n}$ is the unit vector outer normal to $\Gamma_{s i}$ and $\Gamma_{s p}$. These boundary conditions are usually what is measured in the field or in the lab. The time dependence built into the data in (2.7) allows one to model flood and drought seasons.

In addition to the boundary conditions (2.3) and (2.7) imposed along the boundary of the matrix or conduit, respectively, we apply the following interface boundary conditions that couple the solutions in the two domains:

$$
\left.\begin{array}{rl}
\mathbf{v}_{c} \cdot \mathbf{n}_{c m} & =\mathbf{v}_{m} \cdot \mathbf{n}_{c m} \\
-\mathbf{n}_{c m}^{T} \mathbb{T}\left(\mathbf{v}_{c}, p\right) \mathbf{n}_{c m} & =g\left(\phi_{m}-z\right) \\
-\boldsymbol{\tau}_{i}^{T} \mathbb{T}\left(\mathbf{v}_{c}, p\right) \mathbf{n}_{c m} & =\frac{\alpha \nu \sqrt{3}}{\sqrt{\operatorname{trace}(\mathbf{\Pi})}} \boldsymbol{\tau}_{i}^{T}\left(\mathbf{v}_{c}-\mathbf{v}_{m}\right), i=1,2
\end{array}\right\} \quad \text { on } \Gamma_{c m},
$$

where $\left\{\boldsymbol{\tau}_{1}, \boldsymbol{\tau}_{2}\right\}$ represents a local orthonormal basis for the tangent plane to $\Gamma_{c m}, \mathbf{n}_{c m}$ denotes the unit normal to $\Gamma_{c m}$ pointing from the conduit to the matrix, $\mathbb{T}\left(\mathbf{v}_{c}, p\right):=$ $-p \mathbb{I}+2 \nu \mathbb{D}(\mathbf{v})$ denotes the stress tensor, $\alpha$ denotes a constant and $\boldsymbol{\Pi}$ represents the permeability, which has the following relation with the hydraulic conductivity, $\mathbb{K}=$ $\frac{\Pi_{g}}{\nu}$. It should be noticed that $\Pi$ and $\mathbb{K}$ differ by a factor of a constant. Thus, all assumptions on $\mathbb{K}$ such as symmetric positive definiteness also carry over to $\Pi$. In short, $\Pi$ and $\mathbb{K}$ are equivalent in terms of analytical purpose.

The first two interface boundary conditions in (2.8) are quite natural, as we discussed earlier. The first condition guarantees the conservation of mass, i.e., the exchange of fluid between the two domains is conservative. The second condition represents the balance of two driving forces, the kinematic pressure in the matrix and the normal component of the normal stress in the free flow, in the normal direction along the interface.

The last interface equation in (2.8) is the complete form of the well-known Beavers-Joseph condition [8]

$$
\boldsymbol{\tau}_{i}^{T}\left(-2 \nu \mathbb{D}\left(\mathbf{v}_{c}\right)\right) \mathbf{n}_{c m}=\frac{\alpha \nu \sqrt{3}}{\sqrt{\operatorname{trace}(\boldsymbol{\Pi})}} \boldsymbol{\tau}_{1}^{T}\left(\mathbf{v}_{c}-\mathbf{v}_{m}\right), \quad i=1,2
$$

that addresses the important issue of how the porous media affects the conduit flow at the interface. This empirical condition essentially claims that the tangential component of the normal stress that the free flow (the flow part governed by the Stokes equations, i.e., the conduit in our setting.) incurs along the interface is proportional to the jump in the tangential velocity over the interface. Here, $\alpha$ is a parameter depending on the properties of the porous material as well as the geometrical setting of the coupled problem. However, whether the Beavers-Joseph interface condition leads to a well-posed problem is still unclear. Simplified variants of Beavers-Joseph interface conditions are prevalently used, among which the most accepted one is the Beavers-Joseph-Saffman-Jones condition [10,9]. This interface condition drops the term $\boldsymbol{\tau}_{i}^{T}\left(\mathbf{v}_{m}\right)$ on the right hand side and reads as

$$
\boldsymbol{\tau}_{i}^{T}\left(-2 \nu \mathbb{D}\left(\mathbf{v}_{c}\right)\right) \mathbf{n}_{c m}=\frac{\alpha \nu \sqrt{3}}{\sqrt{\operatorname{trace}(\boldsymbol{\Pi})}} \boldsymbol{\tau}_{i}^{T} \mathbf{v}_{c}, \quad i=1,2 .
$$


The interface condition above is used in previous work; see [6]. In [3], the authors omit the whole right hand side of the Beavers-Joseph interface boundary condition. Saffman's simplification is deduced in the case of a simple geometrical setting with a straight interface and statistically one dimensional flows (solutions homogeneous in the direction tangent to the interface in the statistical average). In this specific case suggested by Saffman, under the further assumptions of uniformity of the pressure gradient in the porous medium and the free flow, and the homogeneity of the hydraulic conductivity, the ad hoc asymptotic analysis of the linear Stokes-Darcy model will arrive at the conclusion that, along the interface, the velocity of the porous medium side is a higher-order term compared to the velocity on the conduit flow side as the permeability in the porous medium tends to zero. This simplification is also justified in a more mathematically rigorous way in [11] under a similar setting and assumptions, and the additional hypothesis of periodicity in the horizontal direction. Nevertheless, we will not invoke any of the simplifying assumptions so that we will use the full form of the Beavers-Joseph condition included in (2.8); this will allow us to investigate the reasonableness of various simplifications later.

\subsection{Weak formulation of the time-dependent Stokes-Darcy model.}

From now on we will drop all subscripts (original notation with subscript $c$ denoting conduit, and subscript $m$ denoting matrix) for all functions involved, since the associated domain is always clear within each context.

To define the weak formulation of the coupled problem, we need to define the affine space

$$
\begin{array}{r}
\mathbf{H}_{c, f}:=\left\{\mathbf{w} \in\left(H^{1}\left(\Omega_{c}\right)\right)^{3} \mid \mathbf{w} \cdot \mathbf{n}=f_{s i} \text { on } \Gamma_{s i}, \mathbf{w} \cdot \mathbf{n}=f_{s p} \text { on } \Gamma_{s p}\right. \\
\text { and } \left.\mathbf{w} \times \mathbf{n}=\mathbf{0} \text { on } \Gamma_{s i} \cup \Gamma_{s p}\right\}
\end{array}
$$

and the function spaces

$$
\begin{gathered}
\mathbf{H}_{c, 0}:=\left\{\mathbf{w} \in\left(H^{1}\left(\Omega_{c}\right)\right)^{3} \mid \mathbf{w}=\mathbf{0} \text { on } \Gamma_{s i} \cup \Gamma_{s p}\right\}, \\
H_{p}:=\left\{\varphi \in H^{1}\left(\Omega_{m}\right) \mid \varphi=0 \text { on } \Gamma_{g}\right\} \\
Q:=L^{2}\left(\Omega_{c}\right) \\
\mathbf{W}:=\mathbf{H}_{c, 0} \times H_{p}
\end{gathered}
$$

and

$$
\mathbf{V}:=\mathbf{H}_{c, \operatorname{div}} \times H_{p},
$$

where $\mathbf{H}_{c, \text { div }}:=\left\{\mathbf{w} \in \mathbf{H}_{c, 0} \mid \operatorname{div} \mathbf{w}=0\right\}$. Here, $\mathbf{W}$ and $\mathbf{V}$ are Hilbert spaces with respect to the norm

$$
\|\underline{\mathbf{w}}\|_{\mathbf{W}}:=\frac{1}{\sqrt{2}}\left(\|\mathbf{w}\|_{\mathbf{H}^{1}}^{2}+\|\varphi\|_{H^{1}}^{2}\right)^{1 / 2}, \quad \forall \underline{\mathbf{w}}=(\mathbf{w}, \varphi) \in \mathbf{W} .
$$

On $\Gamma_{c m}$, we consider the trace space (see $[14$, vol. I, p. 66])

$$
\Lambda:=\mathbf{H}_{00}^{1 / 2}\left(\Gamma_{c m}\right) .
$$


This space is a non-closed subspace of $\mathbf{H}^{1 / 2}\left(\Gamma_{c m}\right)$ and has a continuous zero extension to $\mathbf{H}^{1 / 2}\left(\partial \Omega_{c}\right)$. The space $\mathbf{H}_{00}^{1 / 2}\left(\Gamma_{c m}\right)$ could instead be equivalently defined as the restriction of $\mathbf{H}_{c, 0}\left(\Omega_{c}\right)$ to $\Gamma_{c m}$, i.e., $\mathbf{H}_{00}^{1 / 2}\left(\Gamma_{c m}\right)=\left.\mathbf{H}_{c, 0}\left(\Omega_{c}\right)\right|_{\Gamma_{c m}}$. For $\mathbf{H}_{00}^{1 / 2}\left(\Gamma_{c m}\right)$, we have the following continuous imbedding result:

$$
\mathbf{H}_{00}^{1 / 2}\left(\Gamma_{c m}\right) \varsubsetneqq \mathbf{H}_{0}^{1 / 2}\left(\Gamma_{c m}\right)=\mathbf{H}^{1 / 2}\left(\Gamma_{c m}\right) \varsubsetneqq \mathbf{H}^{-1 / 2}\left(\Gamma_{c m}\right) \varsubsetneqq\left(\mathbf{H}_{00}^{1 / 2}\left(\Gamma_{c m}\right)\right)^{\prime}
$$

where $\mathbf{H}_{0}^{1 / 2}\left(\Gamma_{c m}\right)$ is the closure in $\mathbf{H}^{1 / 2}\left(\Gamma_{c m}\right)$ of the space $C_{c}^{\infty}\left(\Gamma_{c m}\right)$ of infinitely differentiable compactly supported functions. In order to understand the dual of $\mathbf{H}_{00}^{1 / 2}\left(\Gamma_{c m}\right)$, we need to note that ${ }^{1}$

$$
\begin{aligned}
\left.\mathbf{H}^{-1 / 2}\left(\partial \Omega_{c}\right)\right|_{\Gamma_{c m}} & \not \mathbf{H}^{-1 / 2}\left(\Gamma_{c m}\right) \text { and } \\
& \left.\mathbf{H}^{-1 / 2}\left(\partial \Omega_{c}\right)\right|_{\Gamma_{c m}} \subset\left(\mathbf{H}_{00}^{1 / 2}\left(\Gamma_{c m}\right)\right)^{\prime} .
\end{aligned}
$$

Henceforth, we use the notational convention that $\underline{\mathbf{u}}=(\mathbf{u}, \phi), \underline{\mathbf{v}}=(\mathbf{v}, \psi)$ and $\underline{\mathbf{w}}=$ $(\mathbf{w}, \varphi)$. They all belong to $\mathbf{W}$.

In order to introduce the weak formulation of the coupled Stokes-Darcy system, we first define the bilinear form $a_{\eta}(\cdot, \cdot): \mathbf{W} \times \mathbf{W} \rightarrow \mathbb{R}$ by

$$
\begin{aligned}
a_{\eta}(\underline{\mathbf{u}}, \underline{\mathbf{v}})= & 2 \nu \int_{\Omega c} \mathbb{D} \mathbf{u}: \mathbb{D} \mathbf{v} \mathrm{d} \Omega_{c}+\frac{\eta}{S} \int_{\Omega_{m}}(\mathbb{K} \nabla \phi) \cdot \nabla \psi \mathrm{d} \Omega_{m} \\
& +g \int_{\Gamma_{c m}} \phi \mathbf{v} \cdot \mathbf{n}_{c m} \mathrm{~d} \Gamma_{c m}-\frac{\eta}{S} \int_{\Gamma_{c m}} \mathbf{u} \cdot \mathbf{n}_{c m} \psi \mathrm{d} \Gamma_{c m} \\
& +\int_{\Gamma_{c m}} \frac{\nu \alpha \sqrt{3}}{\sqrt{\operatorname{trace}(\boldsymbol{\Pi})}} P_{\tau}(\mathbf{u}+\mathbb{K} \nabla \phi) \cdot \mathbf{v d} \Gamma_{c m},
\end{aligned}
$$

$P_{\tau}(\cdot)$ is the projection onto the local tangential plane that can be explicitly defined as $P_{\tau}(\mathbf{v}):=\mathbf{v}-\left(\mathbf{v} \cdot \mathbf{n}_{c m}\right) \mathbf{n}_{c m}$ and where $\eta$ is a scaling parameter that we will exploit in the sequel.

Without further assumptions on the regularity of the domain spaces of $a_{\eta}(\cdot, \cdot)$, we have that $\nabla \phi \in \mathbf{L}^{2}\left(\Omega_{m}\right)$ and thus it does not have a well-defined trace on $\partial \Omega_{m}$ for a general hydraulic conductivity tensor $\mathbb{K}$. Nevertheless, if the hydraulic conductivity is isotropic everywhere, i.e., when the permeability tensor $\mathbf{\Pi}(\mathbf{x})=k(\mathbf{x}) \mathbb{I}$, where $k$ is a scalar function and $\mathbb{I}$ is the identity matrix, then the last term of $a_{\eta}(\cdot, \cdot)$ is well defined in the sense that

$$
\begin{aligned}
\sqrt{3} & \nu \alpha \int_{\Gamma_{c m}} \frac{1}{\sqrt{\operatorname{trace}(\boldsymbol{\Pi})}}\left(P_{\tau}(\mathbf{u})+P_{\tau}(\mathbb{K} \nabla \phi)\right) \cdot P_{\tau}(\mathbf{v}) \mathrm{d} \Gamma_{c m} \\
& =\sqrt{3} \nu \alpha \int_{\Gamma_{c m}} \frac{1}{\sqrt{\operatorname{trace}(\boldsymbol{\Pi})}}\left(P_{\tau}(\mathbf{u})+\frac{g}{\nu} k P_{\tau}(\nabla \phi)\right) \cdot P_{\tau}(\mathbf{v}) \mathrm{d} \Gamma_{c m} \\
& =\nu \alpha \int_{\Gamma_{c m}} \frac{1}{\sqrt{k}} P_{\tau}(\mathbf{u}) \cdot P_{\tau}(\mathbf{v}) \mathrm{d} \Gamma_{c m}+g \alpha \int_{\Gamma_{c m}} \sqrt{k} \nabla_{\tau} \phi \cdot P_{\tau}(\mathbf{v}) \mathrm{d} \Gamma_{c m}
\end{aligned}
$$

where we have used the fact that the tangential projection of the gradient is the tangential derivative of a function defined on the boundary, and the last integral is

\footnotetext{
${ }^{1}$ The space $\left.\mathbf{H}^{-1 / 2}\left(\partial \Omega_{c}\right)\right|_{\Gamma_{c m}}$ is defined in the following way: $\left.\forall f \in \mathbf{H}^{-1 / 2}\left(\partial \Omega_{c}\right)\right|_{\Gamma_{c m}}$ and $g \in$ $\mathbf{H}^{1 / 2}\left(\Gamma_{c m}\right),\left\langle f, g>_{\left.\mathbf{H}^{-1 / 2}\left(\partial \Omega_{c}\right)\right|_{\Gamma_{c m}}, \mathbf{H}^{1 / 2}\left(\Gamma_{c m}\right)}:=<f, \widetilde{g}>_{\mathbf{H}^{-1 / 2}\left(\partial \Omega_{c}\right), \mathbf{H}^{1 / 2}\left(\partial \Omega_{c}\right)}\right.$, where $\widetilde{g}$ is the zero extension of $g$ to $\partial \Omega_{c}$.
} 
understood as an $\left(H_{00}^{1 / 2}\left(\Gamma_{c m}\right)\right)^{\prime}, H_{00}^{1 / 2}\left(\Gamma_{c m}\right)$ duality. More specifically, the gradient of $\phi$ restricted on $\partial \Omega_{m}$ can be represented by $\frac{\partial \phi}{\partial \mathbf{n}} \mathbf{n}+\frac{\partial \phi}{\partial \tau_{1}} \boldsymbol{\tau}_{1}+\frac{\partial \phi}{\partial \tau_{2}} \boldsymbol{\tau}_{2}$, where $\mathbf{n}$ is the local normal direction and $\boldsymbol{\tau}_{1}$ and $\boldsymbol{\tau}_{2}$ are the chosen orthonormal basis of the local tangential plane. Thus, the projection of the gradient to the tangent plane is given by $\frac{\partial \phi}{\partial \tau_{1}} \boldsymbol{\tau}_{1}+\frac{\partial \phi}{\partial \tau_{2}} \boldsymbol{\tau}_{2}$ which is exactly the tangential derivative, i.e., it is the gradient of the function $\left.\phi\right|_{\partial \Omega_{m}}$. Since $\phi \in H_{p}$, we have that $\left.\phi\right|_{\partial \Omega_{m}} \in H^{1 / 2}\left(\partial \Omega_{m}\right)$ and $\nabla_{\tau} \phi\left(\partial \Omega_{m}\right) \in$ $\mathbf{H}^{-1 / 2}\left(\partial \Omega_{m}\right)$. This further implies that $\nabla_{\tau} \phi\left(\Gamma_{c m}\right)=\left.\nabla_{\tau} \phi\left(\partial \Omega_{m}\right)\right|_{\Gamma_{c m}} \in\left(\mathbf{H}_{00}^{-1 / 2}\left(\Gamma_{c m}\right)\right)^{\prime}$, according to $2.12 .^{2}$ We will frequently refer back to this relation in the rest of this work.

Note that the contribution of the Beavers-Joseph term (the last term in (2.13)) is indefinite and of leading order (since, formally, we need $\|\phi\|_{H^{1}}\|\mathbf{v}\|_{H^{1}}$ to bound this term) which is one of the main difficulties in the mathematical analysis. However, if one adopts the simplified Beavers-Joseph-Saffman-Jones interfacial boundary condition, the second part of the last term (which is the indefinite one) drops out and hence the contribution of the simplified interface term to the bilinear form $a_{\eta}(\cdot, \cdot)$ decreases the energy and therefore subsequent analysis is substantially simplified; see $[3,6]$.

We also need to introduce the bilinear form $b(\cdot, \cdot): \mathbf{W} \times Q \rightarrow \mathbb{R}$ associated with the pressure, which is given by

$$
b(\underline{\mathbf{w}}, q):=-\int_{\Omega_{c}} q \nabla \cdot \mathbf{w} \mathrm{d} \Omega_{c},
$$

and the modified duality pairing $<\cdot, \cdot>_{\eta, \mathbf{H}_{c, 0}^{\prime}} \times H_{p}^{\prime}, \mathbf{W}: \mathbf{H}_{c, 0}^{\prime} \times H_{p}^{\prime}, \mathbf{W} \rightarrow \mathbb{R}$ associated with the time derivative given by

$$
<\underline{\mathbf{u}}_{t}, \underline{\mathbf{v}}>_{\eta, \mathbf{H}_{c, 0}^{\prime}} \times H_{p}^{\prime}, \mathbf{W}:=<\mathbf{u}_{t}, \mathbf{v}>_{\mathbf{H}_{c, 0}^{\prime}, \mathbf{H}_{c, 0}}+\eta<\phi_{t}, \psi>_{H_{p}^{\prime}, H_{p}} .
$$

We will use the more economical notation $<\cdot, \cdot>_{\eta}=<\cdot, \cdot>_{\eta, \mathbf{H}_{c, 0}^{\prime}} \times H_{p}^{\prime}, \mathbf{W}$ in the sequel.

REMARK 2.1. Note that the above bilinear forms remain well defined if $\mathbf{u} \in \mathbf{H}_{c, f}$. Actually, this is the affine space in which we want to find the solution of the problem with inhomogeneous Dirichlet boundary conditions at the sinkhole and spring.

Finally, we define the linear forcing functional $F(\cdot): \mathbf{W} \rightarrow \mathbb{R}$ defined by

$$
<F, \underline{\mathbf{w}}>:=-\int_{\Omega_{c}} g \mathbf{k} \cdot \mathbf{w} \mathrm{d} \Omega_{c}+g \int_{\Gamma_{c m}} z \mathbf{w} \cdot \mathbf{n}_{c m} \mathrm{~d} \Gamma,
$$

where $\mathbf{k}$ is the unit vector in $z$ direction and the second term on the right-hand side comes from the second interface condition in (2.8), which is a natural boundary condition.

The weak formulation of the coupled, time-dependent Stokes-Darcy system can be formally derived by multiplying the Stokes system (2.6) by a velocity test function $\mathbf{v}$ then integrating the result over $\Omega_{c}$, multiplying the Darcy equation (2.2) by a scaling parameter $\eta$ and a scalar test function $\psi$ and integrating the result over $\Omega_{m}$, and then taking the sum. Formally, the weak formulation of the coupled time-dependent Stokes-Darcy problem is then given as follows: find $\underline{\mathbf{u}}$ and $p$ such that

$$
\left\{\begin{array}{cc}
<\underline{\mathbf{u}}_{t}, \underline{\mathbf{v}}>_{\eta}+a_{\eta}(\underline{\mathbf{u}}, \underline{\mathbf{v}})+b(\underline{\mathbf{v}}, p)=<F, \underline{\mathbf{v}}> & \forall \underline{\mathbf{v}}=(\mathbf{v}, \psi) \in \mathbf{W} \\
b(\underline{\mathbf{u}}, q)=0 & \forall q \in Q .
\end{array}\right.
$$

\footnotetext{
${ }^{2}$ We could follow another route to reach this conclusion. We know that $\phi\left(\Gamma_{c m}\right) \in H^{1 / 2}\left(\Gamma_{c m}\right)$, then taking the derivative, we have $\nabla_{\tau} \phi\left(\Gamma_{c m}\right) \in\left(\mathbf{H}_{00}^{-1 / 2}\left(\Gamma_{c m}\right)\right)^{\prime}$; see [14].
} 
We further assume that the shapes of $\Gamma_{s i}$ and $\Gamma_{s p}$ are regular enough to guarantee the existence of a continuous extension operator $\mathbf{E}: H^{1 / 2}\left(\Gamma_{s i} \cup \Gamma_{s p}\right) \rightarrow \mathbf{H}_{c, f}\left(\Omega_{c}\right)$ such that $\nabla \cdot \mathbf{E}(f)=0$ and $\mathbf{E}(f) \cdot \mathbf{n}=f$ on $\Gamma_{s i}$ and $\Gamma_{s p}$, where $f=f_{s i}$ and $f=f_{s p}$ on $\Gamma_{s i}$ and $\Gamma_{s p}$, respectively. Then, the solution we seek is $\mathbf{u}-\mathbf{E}(f)$ which belongs to $\mathbf{H}_{c, 0}$. The above weak formulation can be formally rewritten, denoting $(\mathbf{E}(f), 0)$ by $\underline{\underline{\mathbf{u}}}$, as follows: find $\underline{\mathbf{u}}$ and $p$ such that

$$
\left\{\begin{aligned}
<\underline{\mathbf{u}}_{t}, \underline{\mathbf{v}}>_{\eta}+a_{\eta}(\underline{\mathbf{u}}, \underline{\mathbf{v}})+b(\underline{\mathbf{v}}, p) & =<\widetilde{F}, \underline{\mathbf{v}}>_{\eta} & \forall \underline{\mathbf{v}}=(\mathbf{v}, \psi) \in \mathbf{W} \\
b(\underline{\mathbf{u}}, q) & =0 & \forall q \in Q,
\end{aligned}\right.
$$

where the linear functional $\widetilde{F}: \mathbf{W} \rightarrow \mathbb{R}$ is defined by

$$
<\widetilde{F}, \underline{\mathbf{v}}>_{\eta}:=<F, \underline{\mathbf{v}}>-<\widetilde{\mathbf{u}}_{t}, \mathbf{v}>-a_{\eta}(\underline{\widetilde{\mathbf{u}}}, \underline{\mathbf{v}}) \text {. }
$$

The equivalence for smooth solutions between this weak formulation and the classical form can be verified directly; see the appendix.

In order to avoid the difficulty associated with the pressure, we take the LerayHopf approach [15] and look for solutions in the div-free space for $\underline{\mathbf{u}}$ only. More precisely, we look for $\underline{\mathbf{u}} \in L^{2}(0, T ; \mathbf{V}), \underline{\mathbf{u}}_{t}=\underline{\mathbf{u}}^{\prime} \in L^{2}\left(0, T ; \mathbf{V}^{\prime}\right)$ such that

$$
<\underline{\mathbf{u}}_{t}, \underline{\mathbf{v}}>_{\eta}+a_{\eta}(\underline{\mathbf{u}}, \underline{\mathbf{v}})=<\widetilde{F}, \underline{\mathbf{v}}>_{\eta}, \forall \underline{\mathbf{v}}=(\mathbf{v}, \psi) \in \mathbf{V} .
$$

\section{Well-posedness and approximation of the steady-state Stokes-Darcy problem}

In this section, we want to show that the steady-state Stokes-Darcy problem is well-posed (without requiring that the extension $\mathbf{E}(f)$ be div-free) when the coefficient $(\alpha)$ in the Beavers-Joseph interface boundary condition is sufficiently small. Such an assumption is physically relevant since $\alpha$ is expected to scale like the square root of the porosity $n$ (a small quantity for most porous media) [16, 17]. In the steady-state case, the rescaling of the Darcy part is not helpful to the well-posedness. To see this, note that although the rescaled diffusion term could control the indefinite contribution from the Beavers-Joseph interface condition (in the tangential direction), resulting in a Gårding type inequality, in the absence of the time derivative term the rescaling would result in an higher-order indefinite contribution from the term that matches the normal velocities. Thus, the rescaled Darcy equation does not lead to the coercivity of the coupled system. This is also heuristically true because there is no same time scale that we can bring the two different physical problems to in the steady state case. Since the effect of $\eta$ is nullified, we choose $\eta=S g$ to simplify the discussion of the well-posedness of the steady problem. Then, $a_{\eta}(\cdot, \cdot)$ defined in $(2.13)$ reduces to

$$
\begin{aligned}
a(\underline{\mathbf{u}}, \underline{\mathbf{v}})= & 2 \nu \int_{\Omega c} \mathbb{D} \mathbf{u}: \mathbb{D} \mathbf{v} \mathrm{d} \Omega_{c}+g \int_{\Omega_{m}}(\mathbb{K} \nabla \phi) \cdot \nabla \psi \mathrm{d} \Omega_{m} \\
& +g \int_{\Gamma_{c m}} \phi \mathbf{v} \cdot \mathbf{n}_{c m} \mathrm{~d} \Gamma_{c m}-g \int_{\Gamma_{c m}} \mathbf{u} \cdot \mathbf{n}_{c m} \psi \mathrm{d} \Gamma_{c m} \\
& +\int_{\Gamma_{c m}} \frac{\nu \alpha \sqrt{3}}{\sqrt{\operatorname{trace}(\mathbf{\Pi})}} P_{\tau}(\mathbf{u}+\mathbb{K} \nabla \phi) \cdot \mathbf{v} \mathrm{d} \Gamma_{c m}
\end{aligned}
$$

Furthermore, in the steady state case, the isotropy of the hydraulic conductivity is not required by the mathematical treatment in order for the last boundary integral to be well-defined, i.e., we may lift the restriction that requires $\boldsymbol{\Pi}=k(\mathbf{x}) \mathbb{I}$ and let $\mathbf{\Pi}(\mathbf{x})$ 
(and thus $\mathbb{K}(\mathbf{x})$ ) be an arbitrary (location dependent) symmetric, positive definite matrix and the last integral in $a(\cdot, \cdot)$ remains well defined. To see this, first note that, in the steady state case, the Darcy equation becomes $\nabla \cdot(\mathbb{K} \nabla \phi)=0$ which implies that $\mathbb{K} \nabla \phi \in \mathbf{H}_{\text {div }}\left(\Omega_{m}\right):=\left\{\mathbf{w} \in \mathbf{L}^{2}\left(\Omega_{m}\right): \nabla \cdot \mathbf{w} \in L^{2}\left(\Omega_{m}\right)\right\}$; then, by the trace theorem, $(\mathbb{K} \nabla \phi) \cdot \mathbf{n} \in H^{-1 / 2}\left(\partial \Omega_{m}\right)$. But we actually can show that $\mathbb{K} \nabla \phi \in \mathbf{H}^{-1 / 2}\left(\partial \Omega_{m}\right)$. To this end, we decompose $\mathbb{K} \nabla \phi$ as

$$
\mathbb{K} \nabla \phi=\mathbb{K}\left(\frac{\partial \phi}{\partial \mathbf{n}} \mathbf{n}+\frac{\partial \phi}{\partial \tau_{1}} \boldsymbol{\tau}_{1}+\frac{\partial \phi}{\partial \tau_{2}} \boldsymbol{\tau}_{2}\right)
$$

We just need to show $\mathbb{K} \frac{\partial \phi}{\partial \mathbf{n}} \mathbf{n} \in \mathbf{H}^{-1 / 2}\left(\partial \Omega_{m}\right)$. In fact, $\mathbb{K}\left(\frac{\partial \phi}{\partial \tau_{1}} \boldsymbol{\tau}_{1}+\frac{\partial \phi}{\partial \tau_{2}} \boldsymbol{\tau}_{2}\right)$ readily belongs to $\mathbf{H}^{-1 / 2}\left(\partial \Omega_{m}\right)$ when $\mathbb{K}(\mathbf{x})$ is smooth enough, as we argued in the previous section. If the interface is smooth enough, i.e., if $\mathbf{n}(\mathbf{x})$ is a smooth function, we have that

$$
\left(\mathbf{n}^{T} \mathbb{K} \mathbf{n}\right) \frac{\partial \phi}{\partial \mathbf{n}}=\mathbf{n}^{T}\left\{\mathbb{K} \nabla \phi-\mathbb{K}\left(\frac{\partial \phi}{\partial \tau_{1}} \boldsymbol{\tau}_{1}+\frac{\partial \phi}{\partial \tau_{2}} \boldsymbol{\tau}_{2}\right)\right\}=\mathbf{n}^{T} \mathbb{K} \nabla \phi \in H^{-1 / 2}\left(\partial \Omega_{m}\right) .
$$

Now that $\mathbf{n}^{T} \mathbb{K} \mathbf{n}$ is a smooth and strictly positive scalar function, by virtue of the symmetry and positivity of $\mathbb{K}$ and the assumptions on the smoothness of $\mathbb{K}$ and $\mathbf{n}$, we conclude that $\frac{\partial \phi}{\partial \mathbf{n}} \in H^{-1 / 2}\left(\partial \Omega_{m}\right)$. Then, it is straightforward to see that $\left.\nabla \phi\right|_{\partial \Omega_{m}}$ as well as $\left.\mathbb{K} \nabla \phi\right|_{\partial \Omega_{m}}$ belong to $\mathbf{H}^{-1 / 2}\left(\partial \Omega_{m}\right)$. Finally, $\left.\mathbb{K} \nabla \phi\right|_{\Gamma_{m}} \in\left(\mathbf{H}_{00}^{1 / 2}\left(\Gamma_{m}\right)\right)^{\prime}$.

Now that $a(\cdot, \cdot)$ is well defined, we state the weak formulation for the steady state problem: find $\underline{\mathbf{u}} \in \mathbf{W}$ and $p \in Q$ such that:

$$
\left\{\begin{aligned}
a(\underline{\mathbf{u}}, \underline{\mathbf{v}})+b(\underline{\mathbf{v}}, p) & =<F, \underline{\mathbf{v}}>-a(\underline{\widetilde{\mathbf{u}}}, \underline{\mathbf{v}}) & & \forall \underline{\mathbf{v}} \in \mathbf{W} \\
b(\underline{\mathbf{u}}, q) & =-b(\underline{\widetilde{\mathbf{u}}}, q) & & \forall q \in Q,
\end{aligned}\right.
$$

where $\underline{\widetilde{\mathbf{u}}}=(\mathbf{E}(f), 0)$ is the extension of the nonhomogeneous boundary condition. To show the well-posedness, we need to use the well known saddle-point theory [18], i.e., we need to show the following.

1. The bilinear form $a(\cdot, \cdot)$ is $\mathbf{V}$-elliptic, i.e., there exists a constant $\alpha^{3}>0$ such that

$$
a(\underline{\mathbf{v}}, \underline{\mathbf{v}}) \geq \alpha\|\underline{\mathbf{v}}\|_{\mathbf{W}} \quad \forall \underline{\mathbf{v}} \in \mathbf{V}
$$

where the space $\mathbf{V}$ is the defined in (2.10).

2. The bilinear form $b(\cdot, \cdot)$ satisfies the inf-sup condition, i.e., there exists a constant $\beta>0$ such that

$$
\inf _{q \in Q} \sup _{\underline{\mathbf{u}} \in \mathbf{W}} \frac{b(\underline{\mathbf{u}}, q)}{\|\underline{\mathbf{u}}\|_{\mathbf{W}}\|q\|_{L^{2}}} \geq \beta>0 .
$$

We first show that the inf-sup condition holds.

Lemma 3.1. The bilinear functional $b(\cdot, \cdot)$ is continuous on $\mathbf{W} \times Q$ and satisfies the inf-sup condition (3.2).

\footnotetext{
${ }^{3}$ This $\alpha$ is not the same as the parameter in Beavers-Josephs conditions. We will use $\alpha$ to denote both as long as there is no confusion.
} 
Proof. It is obvious that $b(\cdot, \cdot)$ is continuous. In fact,

$$
|b(\underline{\mathbf{u}}, q)|=\left|\int_{\Omega_{c}} q \nabla \cdot \mathbf{u} \mathrm{d} \Omega_{c}\right| \leq\|q\|_{L^{2}}|\mathbf{u}|_{\mathbf{H}^{1}} \leq\|q\|_{L^{2}}\|\mathbf{u}\|_{\mathbf{H}^{1}} \leq\|q\|_{L^{2}}\|\underline{\mathbf{u}}\|_{\mathbf{W}} .
$$

Furthermore, for any $q \in Q$, we can find a $\mathbf{u} \in \mathbf{H}_{c, 0}$ such that

$$
\int_{\Omega_{c}} q \operatorname{div} \mathbf{u d} \Omega_{c} \geq \beta^{*}\|\mathbf{u}\|_{\mathbf{H}^{1}}\|q\|_{L^{2}} \quad \text { with } \beta^{*}>0
$$

see [1]. In our coupled problem, let $\underline{\mathbf{u}}=(-\mathbf{u}, 0)$; then

$$
b(\underline{\mathbf{u}}, q)=\int_{\Omega_{c}} q \operatorname{div} \mathbf{u d} \Omega_{c} \geq \beta^{*}\|q\|_{L^{2}}\|\mathbf{u}\|_{\mathbf{H}^{1}}=\beta\|q\|_{L^{2}}\|\underline{\mathbf{u}}\|_{\mathbf{W}}
$$

with $\beta>0$.

We now move on to show the continuity and coercivity of $a(\cdot, \cdot)$.

Lemma 3.2. The bilinear functional $a(\cdot, \cdot)$ is continuous and coercive on $\mathbf{W} \times \mathbf{W}$ (W-elliptic) when the coefficient in the Beavers-Joseph interface boundary condition $\alpha$ is small enough.

Proof. The continuity is a natural result of the trace theorem and the CauchySchwarz inequality. As for the coercivity, we have

$$
\begin{aligned}
a(\underline{\mathbf{v}}, \underline{\mathbf{v}})= & 2 \nu \int_{\Omega c} \mathbb{D}(\mathbf{v}): \mathbb{D}(\mathbf{v}) \mathrm{d} \Omega_{c}+g \int_{\Omega_{m}}(\mathbb{K} \nabla \psi) \cdot \nabla \psi \mathrm{d} \Omega_{m} \\
& +\nu \alpha \sqrt{3} \int_{\Gamma_{c m}} \frac{1}{\sqrt{\operatorname{trace}(\mathbf{\Pi})}}\left(P_{\tau}(\mathbf{v})+P_{\tau}(\mathbb{K} \nabla \psi)\right) \cdot P_{\tau}(\mathbf{v}) \mathrm{d} \Gamma_{c m} \\
\geq & 2 \nu\|\mathbb{D} \mathbf{v}\|_{\mathbf{L}^{2}}^{2}+g \lambda_{\min }(\mathbb{K})\|\nabla \psi\|_{\mathbf{L}^{2}}^{2}+\frac{\nu \alpha}{\sqrt{\lambda_{\max }(\mathbf{\Pi})}}\left\|P_{\tau}(\mathbf{v})\right\|_{\mathbf{L}^{2}\left(\Gamma_{c m}\right)}^{2} \\
& -\frac{\nu \alpha}{\sqrt{\lambda_{\min }(\mathbf{\Pi})}}\|\mathbb{K} \nabla \psi\|_{\left(\mathbf{H}_{00}^{1 / 2}\left(\Gamma_{c m}\right)\right)^{\prime}}\left\|P_{\tau}(\mathbf{v})\right\|_{\mathbf{H}_{00}^{1 / 2}\left(\Gamma_{c m}\right)} \\
\geq & C_{1} \nu\|\mathbf{v}\|_{\mathbf{H}^{1}}^{2}+C_{2} \lambda_{\min }(\mathbb{K})\|\psi\|_{H^{1}}^{2}-\frac{\nu \alpha \lambda_{\max }(\mathbb{K})}{\sqrt{\lambda_{\min }(\mathbf{\Pi})}}\|\psi\|_{H^{1}\left(\Omega_{m}\right)}\|\mathbf{v}\|_{\mathbf{H}^{1}\left(\Omega_{c}\right)} \\
\geq & \frac{C_{1}}{2} \nu\|\mathbf{v}\|_{\mathbf{H}^{1}}^{2}+\frac{C_{2}}{2} \lambda_{\min }(\mathbb{K})\|\psi\|_{H^{1}}^{2} .
\end{aligned}
$$

Here, the $C_{i}$ 's are strictly positive constants independent of $\mathbb{K}, \nu$, or $\alpha$ and $\mathbb{K}$ is strictly positive definite. The $\lambda_{\max }(\mathbb{K})$ and $\lambda_{\min }(\mathbb{K})$ denote the largest and smallest eigenvalues of $\mathbb{K}$, and $\lambda_{\max }(\boldsymbol{\Pi})$ and $\lambda_{\min }(\boldsymbol{\Pi})$ denote the largest and the smallest eigenvalues of $\Pi$ respectively. The second inequality holds by applying the classical Poincaré inequality, the Poincaré-like inequality in [19, Equ. 4.20], Korn's inequality [20, Thm. 2.4], and the trace theorem and dropping the third term. The last inequality holds if

$$
\left\{\frac{\nu^{\frac{1}{2}} \alpha \lambda_{\max }(\mathbb{K})}{\sqrt{\lambda_{\min }(\mathbb{K})}}\right\}^{2} \leq C_{1} C_{2} \nu \lambda_{\min }(\mathbb{K}) .
$$

This is true when $\alpha^{2}$ is small enough.

REMARK 3.3. If we instead use the Beavers-Joseph-Saffman interface condition for the steady Stokes-Darcy problem, we could obtain, in an easier manner, the coercivity. 
We omit the proof due to the similarity to the proof of the problem considered in [21].

The following result follows from Lemmas 3.1 and 3.2; see, e.g., [22].

Proposition 3.4. The steady-state Stokes-Darcy problem with either the BeaversJoseph (when the coefficient $\alpha$ associated with it is small enough) or Beavers-JosephSaffman interface conditions is well-posed.

We then give an error estimate for the convergence rate of the finite element methods. First, we introduce the following discrete spaces:

$$
\begin{gathered}
\mathbf{W}^{h}=\mathbf{H}_{c, 0}^{h} \times H_{p}^{h} \subset \mathbf{W}, \quad Q^{h} \subset Q \\
\mathbf{V}^{h}=\left\{\underline{\mathbf{v}}^{h} \in \mathbf{W}^{h} \mid b\left(\underline{\mathbf{v}}^{h}, q^{h}\right)=0, \forall q^{h} \in Q^{h}\right\},
\end{gathered}
$$

and

$$
\mathbf{V}_{f}^{h}=\left\{\underline{\mathbf{v}}^{h} \in \mathbf{W}^{h} \mid b\left(\underline{\mathbf{v}}^{h}, q^{h}\right)=-b\left(\underline{\widetilde{\mathbf{u}}}, q^{h}\right), \forall q^{h} \in Q^{h}\right\} .
$$

The spatially discretized problem is to find $\underline{\mathbf{u}}^{h} \in \mathbf{W}^{h}$ and $p^{h} \in Q^{h}$ such that

$$
\left\{\begin{aligned}
a\left(\underline{\mathbf{u}}^{h}, \underline{\mathbf{v}}^{h}\right)+b\left(\underline{\mathbf{v}}^{h}, p^{h}\right) & =<F, \underline{\mathbf{v}}^{h}>-a\left(\underline{\widetilde{\mathbf{u}}}, \underline{\mathbf{v}}^{h}\right) & & \forall \underline{\mathbf{v}}^{h} \in \mathbf{W}^{h} \\
b\left(\underline{\mathbf{u}}^{h}, q^{h}\right) & =-b\left(\underline{\widetilde{\mathbf{u}}}, q^{h}\right) & & \forall q^{h} \in Q^{h} .
\end{aligned}\right.
$$

We assume that the assumptions of Lemma 3.2 are satisfied, i.e., for the discrete case we have

$$
a\left(\underline{\mathbf{v}}^{h}, \underline{\mathbf{v}}^{h}\right) \geq \alpha\left\|\underline{\mathbf{v}}^{h}\right\|_{\mathbf{w}}^{2} \quad \forall \underline{\mathbf{v}}^{h} \in \mathbf{W}^{h},
$$

where $\alpha$ is independent of $h$, and that the finite element spaces satisfy the discrete inf-sup or div-stability condition

$$
\inf _{0 \neq q^{h} \in Q^{h}} \sup _{0 \neq \underline{\mathbf{v}}^{h} \in \mathbf{W}^{h}} \frac{b\left(\underline{\mathbf{v}}^{h}, q^{h}\right)}{\left\|\underline{\mathbf{v}}^{h}\right\| \mathbf{W}\left\|q^{h}\right\|_{L^{2}}} \geq \beta>0 \quad \forall h .
$$

Proposition 3.5. Under the above assumptions of coercivity and div-stability, we have the following error estimate for the solution of problem (3.3):

$$
\left\|\underline{\mathbf{u}}-\underline{\mathbf{u}}^{h}\right\| \mathbf{w}+\left\|p-p^{h}\right\|_{L^{2}} \leq C\left(\inf _{\underline{\mathbf{v}}^{h} \in \mathbf{W}^{h}}\left\|\underline{\mathbf{u}}-\underline{\mathbf{v}}^{h}\right\| \mathbf{W}+\inf _{q^{h} \in Q^{h}}\left\|p-q^{h}\right\|_{L^{2}}\right),
$$

where $\underline{\mathbf{u}}$ is the solution of problem (3.1).

Proof. Given in the appendix.

REMARK 3.6. If the unique solution pair $(\phi, \xi)$ of the adjoint problem

$$
\left\{\begin{aligned}
a\left(\underline{\mathbf{v}}^{h}, \phi\right)+b\left(\underline{\mathbf{v}}^{h}, \xi\right) & =<e, \underline{\mathbf{v}}^{h}>_{\mathbf{L}^{2} \times L^{2}, \mathbf{L}^{2} \times L^{2}} & & \forall \underline{\mathbf{v}}^{h} \in \mathbf{W}^{h} \\
b\left(\phi, q^{h}\right) & =0 & & \forall q^{h} \in Q^{h},
\end{aligned}\right.
$$

where $e=\underline{\mathbf{u}}-\underline{\mathbf{u}}^{h}$, is sufficiently regular, then by the classical duality argument (see [22, pp. 119-120]) we have the estimate for the error $e$ in $\mathbf{L}^{2} \times L^{2}$ given by

$$
\left\|\underline{\mathbf{u}}-\underline{\mathbf{u}}^{h}\right\|_{\mathbf{L}^{2} \times L^{2}} \leq C h\left(\left\|\underline{\mathbf{u}}-\underline{\mathbf{u}}^{h}\right\|_{\mathbf{W}}+\left\|p-p^{h}\right\|_{L^{2}}\right) .
$$




\section{The time dependent coupled Stokes-Darcy problem}

Although the steady-state problem does provide some practical insights, stationary phenomena in the types of flows we are interested in are rare compared with transient ones. Many common factors drive practical aquifer flows to be time dependent. For instance, seasonal precipitation is a prevalent time-dependent factor that dominantly affects the groundwater flows. The well-posedness of the coupled StokesDarcy model with the Beavers-Joseph interface boundary condition poses difficulties even for isotropic hydraulic conductivity $\mathbb{K}$, i.e., when $\mathbb{K}=\frac{g}{\nu} k(\mathbf{x}) \mathbb{I}$. However, in the transient case, the time derivative term together with the dissipative terms enable us to control the interfacial term which leads to the well-posedness.

To this end, we recall the weak formulation (2.16) which is derived by adding the Stokes system (2.6) and $\eta$ times the Darcy system (2.2) and homogenizing the boundary condition at $\Gamma_{s i}$ and $\Gamma_{s p}$ with the re-scaling parameter $\eta$ at our disposal. Here, we will further exploit this parameter. Indeed, we will show that for large enough $\eta$, the bilinear term (2.13) is essentially coercive in the sense of satisfying a Gårding type inequality (4.2) under the assumption that we have isotropic ${ }^{4}$ (but not necessarily homogeneous) porous media, i.e., $\mathbb{K}(\mathbf{x})=\frac{g}{\nu} \boldsymbol{\Pi}=\frac{g}{\nu} k(\mathbf{x}) \mathbb{I}$. This essentially leads to the well-posedness. In retrospect, the choice of a large rescaling parameter $\eta$ makes sense since the flow in porous media evolves on a relatively slow time scale compared to that of the flow in the conduit, and the re-scaling will essentially bring them to the same time scale for easy comparison.

With eventual full discretization involving finite element approximation in mind, we approximate (2.15) instead of (2.16) via a backward-Euler discretization in time. Letting $\delta=\Delta t$, we have the semi-discrete system for $\underline{\mathbf{u}}^{m} \in \mathbf{W}$ and $p^{m} \in L^{2}\left(\Omega_{c}\right)$

$$
\left\{\begin{aligned}
\frac{1}{\delta}\left\langle\left(\begin{array}{c}
\mathbf{u}^{m+1}-\mathbf{u}^{m} \\
\eta\left(\phi^{m+1}-\phi^{m}\right)
\end{array}\right), \underline{\mathbf{v}}\right\rangle+a_{\eta}\left(\underline{\mathbf{u}}^{m+1}, \underline{\mathbf{v}}\right) & \\
+b\left(\underline{\mathbf{v}}, p^{m+1}\right)=<F^{m}, \underline{\mathbf{v}}> & \forall \underline{\mathbf{v}} \in \mathbf{W} \\
b\left(\underline{\mathbf{u}}^{m+1}, q\right)=0 & \forall q \in Q,
\end{aligned}\right.
$$

where

$$
F^{m}=\frac{1}{\delta} \int_{m \delta}^{(m+1) \delta} \widetilde{F}(t) \mathrm{d} t
$$

This scheme may be also viewed as a time discretization of the div-free formulation (2.16) when we take $\underline{\mathbf{u}}^{m}, \mathbf{v} \in \mathbf{V}$.

We may rewrite the first equation in (4.1) as

$$
\begin{aligned}
& \frac{1}{\delta}\left\langle\left(\begin{array}{c}
\mathbf{u}^{m+1} \\
\eta \phi^{m+1}
\end{array}\right), \underline{\mathbf{v}}\right\rangle+a_{\eta}\left(\underline{\mathbf{u}}^{m+1}, \underline{\mathbf{v}}\right)+b\left(\underline{\mathbf{v}}, p^{m+1}\right) \\
= & <F^{m}, \underline{\mathbf{v}}>+\frac{1}{\delta}\left\langle\left(\begin{array}{c}
\mathbf{u}^{m} \\
\eta \phi^{m}
\end{array}\right), \underline{\mathbf{v}}\right\rangle \quad \forall \underline{\mathbf{v}} \in \mathbf{W}
\end{aligned}
$$

and denote the sum of the first two terms on the left-hand side of the above equation by $a_{\delta, \eta}\left(\underline{\mathbf{u}}^{m+1}, \underline{\mathbf{v}}\right)$, i.e.,

$$
a_{\delta, \eta}(\underline{\mathbf{u}}, \underline{\mathbf{v}}):=\frac{1}{\delta}\left\langle\left(\begin{array}{c}
\mathbf{u} \\
\eta \phi
\end{array}\right), \underline{\mathbf{v}}\right\rangle+a_{\eta}(\underline{\mathbf{u}}, \underline{\mathbf{v}})
$$

\footnotetext{
${ }^{4}$ The isotropy assumption is not needed if we use the Beavers-Joseph-Saffman-Jones interface boundary condition.
} 
In order to show the solvability of $\underline{\mathbf{u}}^{m+1}$, we again do the same as we did in the last section, i.e., we invoke the general theory for saddle-point problems. For the bilinear form $b(\cdot, \cdot)$, both the inf-sup condition (Lemma 3.1) and its continuity are verified in the last section. It remains to show that $a_{\delta, \eta}(\cdot, \cdot)$ is continuous and $\mathbf{V}$-elliptic. In fact, we are going to show a stronger result, namely that it is $\mathbf{W}$-elliptic.

Firstly, it is obvious that $a_{\delta, \eta}(\underline{\mathbf{u}}, \underline{\mathbf{v}})$ is bilinear and continuous. In fact, when the hydraulic conductivity is isotropic, we have

$$
\begin{aligned}
& \quad\left|a_{\delta, \eta}(\underline{\mathbf{u}}, \underline{\mathbf{v}})\right| \\
& \leq C_{1}\left(\|\mathbb{D} \mathbf{u}\|_{L^{2}}\|\mathbb{D} \mathbf{v}\|_{L^{2}}+\|\nabla \phi\|_{L^{2}}\|\nabla \psi\|_{L^{2}}\right. \\
& \quad+\|\phi\|_{L^{2}\left(\Gamma_{c m}\right)}\|\mathbf{v} \cdot \mathbf{n}\|_{L^{2}\left(\Gamma_{c m}\right)}+\|\psi\|_{L^{2}\left(\Gamma_{c m}\right)}\|\mathbf{u} \cdot \mathbf{n}\|_{L^{2}\left(\Gamma_{c m}\right)} \\
& \left.\quad+\|\mathbf{u}\|_{L^{2}\left(\Gamma_{c m}\right)}\|\mathbf{v}\|_{L^{2}\left(\Gamma_{c m}\right)}+\left\|\nabla_{\tau} \phi\right\|_{\left(\mathbf{H}_{00}^{1 / 2}\left(\Gamma_{c m}\right)\right)^{\prime}}\|\mathbf{v}\|_{\mathbf{H}_{00}^{1 / 2}\left(\Gamma_{c m}\right)}\right) \\
& \leq C_{2}\left(\|\underline{\mathbf{u}}\|_{\mathbf{W}}\|\underline{\mathbf{v}}\|_{\mathbf{W}}+\|\phi\|_{\mathbf{H}^{1 / 2}\left(\partial \Omega_{m}\right)}\|\mathbf{v}\|_{\mathbf{H}^{1 / 2}\left(\partial \Omega_{c}\right)}\right) \leq C_{3}\|\underline{\mathbf{u}}\|_{\mathbf{W}}\|\underline{\mathbf{v}}\|_{\mathbf{W}},
\end{aligned}
$$

where $C_{1}, C_{2}$, and $C_{3}$ are generic constants independent of the unknown functions. Therefore, $a_{\delta, \eta}(\cdot, \cdot)$ is continuous on $\mathbf{W} \times \mathbf{W}$.

As for the coercivity (the $\mathbf{W}$-ellipticity), we have, thanks to the Korn and Poincaré inequalities and various trace estimates ${ }^{5}$ :

$$
\begin{aligned}
& a_{\delta, \eta}(\underline{\mathbf{u}}, \underline{\mathbf{u}}) \\
= & \frac{1}{\delta}\left(\|\mathbf{u}\|_{L^{2}\left(\Omega_{c}\right)}^{2}+\eta\|\phi\|_{L^{2}\left(\Omega_{m}\right)}^{2}\right)+2 \nu\|\mathbb{D} \mathbf{u}\|_{L^{2}\left(\Omega_{c}\right)}^{2} \\
& +\frac{\eta g}{S \nu} \int_{\Omega_{m}} k \nabla \phi \cdot \nabla \phi \mathrm{d} \Omega_{m}+\left(g-\frac{\eta}{S}\right) \int_{\Gamma_{c m}} \phi \mathbf{u} \cdot \mathbf{n} \mathrm{d} \Gamma \\
& +\left\|\sqrt{\frac{\alpha \nu}{\sqrt{k}}} P_{\tau}(\mathbf{u})\right\|_{L^{2}\left(\Gamma_{c m}\right)}^{2}+<\alpha g \sqrt{k} \nabla_{\tau} \phi, P_{\tau} \mathbf{u}>\left(\mathbf{H}_{\mathrm{oo}}^{1 / 2}\left(\Gamma_{c m}\right)\right)^{\prime}, \mathbf{H}_{00}^{1 / 2}\left(\Gamma_{c m}\right) \\
\geq & \frac{1}{\delta}\left(\|\mathbf{u}\|_{L^{2}\left(\Omega_{c}\right)}^{2}+\eta\|\phi\|_{L^{2}\left(\Omega_{m}\right)}^{2}\right)+2 \nu C_{1}\|\nabla \mathbf{u}\|_{L^{2}\left(\Omega_{c}\right)}^{2} \\
& +\frac{\eta g C_{k, 1}}{S \nu}\|\nabla \phi\|_{L^{2}\left(\Omega_{m}\right)}^{2}-\left|\frac{\eta}{S}-g\right|\|\phi\|_{L^{2}\left(\Gamma_{c m}\right)}\|\mathbf{u} \cdot \mathbf{n}\|_{L^{2}\left(\Gamma_{c m}\right)} \\
& +\frac{\sqrt{3} \alpha \nu}{C_{k, 2}}\left\|P_{\tau}(\mathbf{u})\right\|_{L^{2}\left(\Gamma_{c m}\right)}^{2}-\alpha g C_{k, 3}\|\phi\|_{\mathbf{H}^{1 / 2}\left(\partial \Omega_{m}\right)}\|\mathbf{u}\|_{\mathbf{H}^{1 / 2}\left(\partial \Omega_{c}\right)} \\
\geq & \frac{1}{\delta}\left(\|\mathbf{u}\|_{L^{2}\left(\Omega_{c}\right)}^{2}+\eta\|\phi\|_{L^{2}\left(\Omega_{m}\right)}^{2}\right)+2 \nu C_{1}\|\nabla \mathbf{u}\|_{L^{2}\left(\Omega_{c}\right)}^{2}+\frac{\eta g C_{k, 1}}{S \nu}\|\nabla \phi\|_{L^{2}\left(\Omega_{m}\right)}^{2} \\
& -\left(\frac{\eta}{S}+g\right) C_{2}\|\phi\|_{L^{2}\left(\Omega_{m}\right)}^{1 / 2}\|\nabla \phi\|_{L^{2}\left(\Omega_{m}\right)}^{1 / 2}\|\mathbf{u}\|_{L^{2}\left(\Omega_{c}\right)}^{1 / 2}\|\nabla \mathbf{u}\|_{L^{2}\left(\Omega_{c}\right)}^{1 / 2} \\
& -C_{3} \alpha g C_{k, 3}\|\nabla \phi\|_{L^{2}\left(\Omega_{m}\right)}\|\nabla \mathbf{u}\|_{L^{2}\left(\Omega_{c}\right)} \\
\geq & \frac{1}{\delta}\|\mathbf{u}\|_{L^{2}\left(\Omega_{c}\right)}^{2}+\frac{\eta}{\delta}\|\phi\|_{L^{2}\left(\Omega_{m}\right)}^{2}+2 \nu C_{1}\|\nabla \mathbf{u}\|_{L^{2}\left(\Omega_{c}\right)}^{2}+\frac{\eta g C_{k, 1}}{S \nu}\|\nabla \phi\|_{L^{2}\left(\Omega_{m}\right)}^{2} \\
& -\frac{\nu C_{1}}{2}\|\nabla \mathbf{u}\|_{L^{2}\left(\Omega_{c}\right)}^{2}-\frac{\eta g C_{k, 1}}{4 S \nu}\|\nabla \phi\|_{L^{2}\left(\Omega_{m}\right)}^{2}
\end{aligned}
$$

\footnotetext{
${ }^{5}$ One trace inequality used here is $\|u\|_{L^{2}\left(\Gamma_{c m}\right)}^{2} \leq C\|u\|_{L^{2}(\Omega)}\|u\|_{H^{1}(\Omega)}$, which can be verified easily using the calculus identity $f^{2}(0)=f^{2}(x)-2 \int_{0}^{x} f(s) f^{\prime}(s) \mathrm{d} s$.
} 


$$
\begin{aligned}
& -\frac{S^{1 / 2}\left(\frac{\eta}{S}+g\right)^{2} C_{2}^{2}}{\left(C_{k, 1} g \eta C_{1}\right)^{1 / 2}}\|\phi\|_{L^{2}\left(\Omega_{m}\right)}\|\mathbf{u}\|_{L^{2}\left(\Omega_{c}\right)} \\
& -\frac{\nu C_{1}}{2}\|\nabla \mathbf{u}\|_{L^{2}\left(\Omega_{c}\right)}^{2}-\frac{\left(C_{3} \alpha C_{k, 3}\right)^{2} g^{2}}{C_{1} \nu}\|\nabla \phi\|_{L^{2}\left(\Omega_{m}\right)}^{2},
\end{aligned}
$$

where the $C_{i}$ 's are generic constants depending on the geometry of the domain but independent of the other parameters such as $k, \eta, \nu, S, g, \alpha$, and $\delta$. The $C_{k, i}$ 's are constants related to the permeability $k$. Roughly speaking, $C_{k, 1}$ is proportional to $k$, while $C_{k, 2}$ and $C_{k, 3}$ are proportional to $\sqrt{k}$. These constants are obtainable by virtue of the smoothness of $k$.

Now, it is easy to see that $a_{\delta, \eta}(\cdot, \cdot)$ is coercive for small enough $\delta$ and large enough $\eta$. Indeed, with all other parameters fixed, we may choose the time step $\delta$ small enough and the rescaling parameter $\eta$ large enough so that the following inequalities hold:

$$
\left\{\begin{aligned}
\frac{\eta g C_{k, 1}}{4 S \nu} & \geq \frac{\left(C_{3} \alpha C_{k, 3}\right)^{2} g^{2}}{C_{1} \nu} \\
\frac{\eta}{\delta^{2}} & \geq\left(\frac{S^{1 / 2}}{\left(C_{k, 1} g \eta C_{1}\right)^{1 / 2}}\left(\frac{\eta}{S}+g\right)^{2} C_{2}^{2}\right)^{2} .
\end{aligned}\right.
$$

Then, we have the coercivity of $a_{\delta, \eta}$ :

$$
\begin{aligned}
a_{\delta, \eta}(\underline{\mathbf{u}}, \underline{\mathbf{u}}) \geq \frac{1}{2 \delta}\|\mathbf{u}\|_{L^{2}\left(\Omega_{c}\right)}^{2} & +\frac{\eta}{2 \delta}\|\phi\|_{L^{2}\left(\Omega_{m}\right)}^{2} \\
& +\frac{\nu C_{1}^{2}}{2}\|\nabla \mathbf{u}\|_{L^{2}\left(\Omega_{c}\right)}^{2}+\frac{\eta g C_{k, 1}}{2 S \nu}\|\nabla \phi\|_{L^{2}\left(\Omega_{m}\right)}^{2} .
\end{aligned}
$$

Therefore we have established the existence of the discrete problem (4.1).

As a byproduct, we have also derived a Gårding type inequality indicating that $a_{\eta}$ is essentially coercive in the sense that there exists $C_{0}>0$ and $\alpha_{\eta}>0$ such that $a_{\eta}(\underline{\mathbf{u}}, \underline{\mathbf{v}})+C_{0}(\underline{\mathbf{u}}, \underline{\mathbf{v}})$ is coercive, i.e.,

$$
a_{\eta}(\underline{\mathbf{u}}, \underline{\mathbf{u}}) \geq \alpha_{\eta}\|\underline{\mathbf{u}}\|_{\mathbf{W}}^{2}-C_{0}\|\underline{\mathbf{u}}\|_{\mathbf{L}^{2} \times L^{2}}^{2} .
$$

Our next goal is to show that the solutions of the backward-Euler scheme converge to a solution to the weak formulation (2.15). We start with the derivation of a priori estimates for the approximate solutions. We estimate $\underline{\mathbf{u}}^{m+1}$ by using the fact (see for instance $[23,15])$ that the solution to $(4.1)$ is also the unique solution to the following problem: find $\underline{\mathbf{u}}^{m+1}$ in $\mathbf{V}$ such that

$$
\frac{1}{\delta}\left\langle\left(\begin{array}{c}
\mathbf{u}^{m+1}-\mathbf{u}^{m} \\
\eta\left(\phi^{m+1}-\phi^{m}\right)
\end{array}\right), \underline{\mathbf{v}}\right\rangle+a_{\eta}\left(\underline{\mathbf{u}}^{m+1}, \underline{\mathbf{v}}\right)=<F^{m}, \underline{\mathbf{v}}>\quad \forall \underline{\mathbf{v}} \in \mathbf{V} .
$$

Setting $\underline{\mathbf{v}}=\left(\begin{array}{c}\mathbf{u}^{m+1} \\ \phi^{m+1}\end{array}\right)$ in (4.3) and using the identity $(a-b, a)=\frac{1}{2}\left(|a|^{2}-|b|^{2}+|a-b|^{2}\right)$, we have

$$
\begin{gathered}
\quad\left\|\mathbf{u}^{m+1}\right\|_{L^{2}}^{2}-\left\|\mathbf{u}^{m}\right\|_{L^{2}}^{2}+\left\|\mathbf{u}^{m+1}-\mathbf{u}^{m}\right\|_{L^{2}}^{2} \\
\quad+\eta\left(\left\|\phi^{m+1}\right\|_{L^{2}}^{2}-\left\|\phi^{m}\right\|_{L^{2}}^{2}+\left\|\phi^{m+1}-\phi^{m}\right\|_{L^{2}}^{2}\right)+2 \delta a_{\eta}\left(\underline{\mathbf{u}}^{m+1}, \underline{\mathbf{u}}^{m+1}\right) \\
=2 \delta\left(F^{m}, \underline{\mathbf{u}}^{m+1}\right) \leq 2 \delta\left\|F^{m}\right\|_{\mathbf{V}^{\prime}}\left\|\underline{\mathbf{u}}^{m+1}\right\|_{\mathbf{V}} .
\end{gathered}
$$


Hence,

$$
\begin{aligned}
& \left\|\mathbf{u}^{m+1}\right\|_{L^{2}}^{2}-\left\|\mathbf{u}^{m}\right\|_{L^{2}}^{2}+\left\|\mathbf{u}^{m+1}-\mathbf{u}^{m}\right\|_{L^{2}}^{2} \\
& +\eta\left(\left\|\phi^{m+1}\right\|_{L^{2}}^{2}-\left\|\phi^{m}\right\|_{L^{2}}^{2}+\left\|\phi^{m+1}-\phi^{m}\right\|_{L^{2}}^{2}\right)+\alpha_{\eta} \delta\left\|\underline{\mathbf{u}}^{m+1}\right\|_{\mathbf{V}}^{2} \\
& \leq 2 \delta C_{0}\left\|\underline{\mathbf{u}}^{m+1}\right\|_{L^{2}}^{2}+\frac{\delta}{\alpha_{\eta}}\left\|F^{m}\right\|_{\mathbf{V}^{\prime}}^{2},
\end{aligned}
$$

where $C_{0}$ is independent of $\delta$, provided $\delta$ is small enough. Summing from $m=0$ to $N-1$, with $T:=N \delta=N \Delta t$, we have

$$
\begin{gathered}
\left\|\mathbf{u}^{N}\right\|_{L^{2}}^{2}+\sum_{m=0}^{N-1}\left\|\mathbf{u}^{m+1}-\mathbf{u}^{m}\right\|_{L^{2}}^{2} \\
+\eta\left(\left\|\phi^{N}\right\|_{L^{2}}^{2}+\sum_{m=0}^{N-1}\left\|\phi^{m+1}-\phi^{m}\right\|_{L^{2}}^{2}\right)+\alpha_{\eta} \delta \sum_{m=0}^{N-1}\left\|\underline{\mathbf{u}}^{m+1}\right\|_{\mathbf{V}}^{2} \\
\leq 2 \delta C_{0} \sum_{m=0}^{N-1}\left\|\underline{\mathbf{u}}^{m+1}\right\|_{L^{2}}^{2}+\frac{\delta}{\alpha_{\eta}} \sum_{m=0}^{N-1}\left\|F^{m}\right\|_{\mathbf{V}^{\prime}}^{2}+\left\|\mathbf{u}^{0}\right\|_{L^{2}}^{2}+\eta\left\|\phi^{0}\right\|_{L^{2}}^{2} \\
\leq 2 \delta C_{0} \sum_{m=0}^{N-1}\left\|\underline{\mathbf{u}}^{m+1}\right\|_{L^{2}}^{2}+\left\|\mathbf{u}^{0}\right\|_{L^{2}}^{2}+\eta\left\|\phi^{0}\right\|_{L^{2}}^{2}+\frac{\delta}{\alpha_{\eta}} \int_{0}^{T}\|\widetilde{F}(s)\|_{\mathbf{V}^{\prime}}^{2} \mathrm{~d} s .
\end{gathered}
$$

Therefore, we have the following a priori estimates

$$
\left\{\begin{aligned}
\sum_{m=0}^{N-1}\left\|\underline{\mathbf{u}}^{m+1}-\underline{\mathbf{u}}^{m}\right\|_{\mathbf{L}^{2} \times L^{2}} & \leq C \\
\left\|\underline{\mathbf{u}}^{m}\right\|_{\mathbf{L}^{2} \times L^{2}} & \leq C \quad \text { for } 1 \leq m \leq N \\
\delta \sum_{m=0}^{N-1}\left\|\underline{\mathbf{u}}^{m+1}\right\|_{\mathbf{V}}^{2} & \leq C,
\end{aligned}\right.
$$

where $C$ is a constant independent of $m$ and we have applied the discrete Gronwall type inequality, which states that if $y_{n} \leq A+B \delta \sum_{j=0}^{n-1} y_{j}$ for $1 \leq n \leq N$ and $\delta=T / N$, then $\max _{1 \leq j \leq N} y_{j} \leq A e^{B T}$.

Furthermore, by using the inf-sup condition and (4.1), we have an estimate for the pressure at each time step. For each $p^{m+1}$, there exists a $\underline{\mathbf{v}}^{m+1}$ such that

$$
\begin{gathered}
\beta\left\|p^{m+1}\right\|_{L^{2}}\left\|\underline{\mathbf{v}}^{m+1}\right\|_{\mathbf{W}} \leq b\left(\underline{\mathbf{v}}^{m+1}, p^{m+1}\right) \\
\leq\left|\frac{1}{\delta}<\mathbf{u}^{m+1}-\mathbf{u}^{m}, \mathbf{v}^{m+1}>+\frac{\eta}{\delta}<\phi^{m+1}-\phi^{m}, \psi^{m+1}>\right| \\
\quad+\left|a_{\eta}\left(\underline{\mathbf{u}}^{m+1}, \underline{\mathbf{v}}^{m+1}\right)\right|+\left|<F^{m}, \underline{\mathbf{v}}^{m+1}>\right| \\
\leq 2\left\|\frac{1}{\delta}\left(\begin{array}{c}
\mathbf{u}^{m+1} \\
\eta\left(\phi^{m+1}\right)
\end{array}\right)\right\|_{\mathbf{L}^{2} \times L^{2}}\left\|\underline{\mathbf{v}}^{m+1}\right\|_{\mathbf{L}^{2} \times L^{2}} \\
\quad+C\left\|\underline{\mathbf{u}}^{m+1}\right\|_{\mathbf{W}}\left\|\underline{\mathbf{v}}^{m+1}\right\|_{\mathbf{W}}+\left\|F^{m}\right\|_{\mathbf{W}^{\prime}}\left\|\underline{\mathbf{v}}^{m+1}\right\|_{\mathbf{W}} .
\end{gathered}
$$

Hence,

$$
\left\|p^{m+1}\right\|_{L^{2}} \leq C_{\eta}\left(\frac{1}{\delta}\left\|\left(\begin{array}{c}
\mathbf{u}^{m+1} \\
\eta\left(\phi^{m+1}\right)
\end{array}\right)\right\|_{\mathbf{L}^{2} \times L^{2}}+\left\|\underline{\mathbf{u}}^{m+1}\right\|_{\mathbf{W}}+\left\|F^{m}\right\|_{\mathbf{W}^{\prime}}\right) .
$$

However, we note that the $\left\{p^{m}\right\}$ may not be uniformly bounded in $L^{2}$ as $\delta \rightarrow 0$. 
Next, we define two approximate solutions for $\underline{\mathbf{u}}$ on $[0, T], T=N \delta$ :

$$
\underline{\mathbf{u}}_{\delta}^{*}((m+1) \delta)=\underline{\mathbf{u}}^{m+1}, \quad \underline{\mathbf{u}}_{\delta}^{*} \text { piecewise linear on }[0, T],
$$

i.e., $\underline{\mathbf{u}}_{\delta}^{*}$ is linear on $(m \delta,(m+1) \delta]$, and

$$
\underline{\mathbf{u}}_{\delta}^{* *}((m+1) \delta)=\underline{\mathbf{u}}^{m+1}, \quad \underline{\mathbf{u}}_{\delta}^{* *} \text { piecewise constant on }[0, T],
$$

i.e., $\underline{\mathbf{u}}_{\delta}^{* *}$ is constant on $(m \delta,(m+1) \delta]$, and one approximate solution for $p$ :

$$
p_{\delta}^{* *}((m+1) \delta)=p^{m+1}, \quad p_{\delta}^{* *} \text { piecewise constant on }[0, T] .
$$

We may then rewrite (4.1) as,

$$
\begin{aligned}
\left\langle\left(\begin{array}{c}
\frac{\mathrm{d} \mathbf{u}_{\delta}^{*}}{\mathrm{~d} t} \\
\eta \frac{\mathrm{d} \phi_{\delta}^{*}}{\mathrm{~d} t}
\end{array}\right), \underline{\mathbf{v}}\right\rangle+a_{\eta}\left(\underline{\mathbf{u}}_{\delta}^{* *}, \underline{\mathbf{v}}\right)+b\left(\underline{\mathbf{v}}, p_{\delta}^{* *}\right) & =<F^{m}(t), \underline{\mathbf{v}}> \\
b\left(\underline{\mathbf{u}}_{\delta}^{*}, q\right) & =0 .
\end{aligned}
$$

The a priori estimates we derived imply that

$$
\underline{\mathbf{u}}_{\delta}^{*}, \underline{\mathbf{u}}_{\delta}^{* *} \in L^{2}(0, T ; \mathbf{V}), \quad \underline{\mathbf{u}}_{\delta}^{*^{\prime}} \in L^{2}\left(0, T ; \mathbf{V}^{\prime}\right), \quad \text { and } \quad \underline{\mathbf{u}}_{\delta}^{*}, \underline{\mathbf{u}}_{\delta}^{* *} \in L^{\infty}\left(0, T ; \mathbf{L}^{2} \times L^{2}\right)
$$

are uniformly bounded independent of $\delta$. Therefore, we may extract a sub-sequence (without changing the notation) such that

$$
\underline{\mathbf{u}}_{\delta}^{*} \underset{\delta \rightarrow 0}{\stackrel{w}{\longrightarrow}} \underline{\mathbf{u}}^{(1)}, \underline{\mathbf{u}}^{(1)} \quad \text { and } \quad \underline{\mathbf{u}}_{\delta}^{* *} \underset{\delta \rightarrow 0}{\stackrel{w}{\longrightarrow}} \underline{\mathbf{u}}^{(2)}
$$

weakly in $L^{2}(0, T ; \mathbf{V})$,

$$
\underline{\mathbf{u}}_{\delta}^{*^{\prime}} \underset{\delta \rightarrow 0}{\stackrel{w}{\longrightarrow}} \underline{\mathbf{w}}^{*}
$$

weakly in $L^{2}\left(0, T ; \mathbf{V}^{\prime}\right)$ and

$$
\underline{\mathbf{u}}_{\delta}^{*} \underset{\delta \rightarrow 0}{\stackrel{w^{*}}{\longrightarrow}} \underline{\mathbf{u}}^{(1)} \quad \text { and } \quad \underline{\mathbf{u}}_{\delta}^{* *} \underset{\delta \rightarrow 0}{\stackrel{w^{*}}{\longrightarrow}} \underline{\mathbf{u}}^{(2)}
$$

weak * in $L^{\infty}\left(0, T ; \mathbf{L}^{2} \times L^{2}\right)$.

It is easy to see that $\underline{\mathbf{u}}^{(1)}=\underline{\mathbf{u}}^{(2)}=\underline{\mathbf{u}}=(\mathbf{u}, \phi)$ (see (4.6)) and $\underline{\mathbf{w}}^{*}=\underline{\mathbf{u}}^{\prime}$ which implies that

$$
\underline{\mathbf{u}} \in L^{2}(0, T ; \mathbf{V}), \quad \underline{\mathbf{u}}^{\prime} \in L^{2}\left(0, T ; \mathbf{V}^{\prime}\right) .
$$

We also have $F_{\delta}(t) \rightarrow \widetilde{F}(t)$ in $L^{2}\left(0, T ; \mathbf{V}^{\prime}\right)$, where

$$
F_{\delta}(t)=\frac{1}{\delta} \int_{m \delta}^{(m+1) \delta} \widetilde{F}(s) \mathrm{d} s \quad \text { with } t \in[m \delta,(m+1) \delta] .
$$

Now we derive the uniform a priori estimates on the pressure by utilizing the 
approximation equation and the a priori estimate for $\underline{\mathbf{u}}^{*}$ and $\underline{\mathbf{u}}^{* *}$. Indeed,

$$
\begin{aligned}
& \left\|p_{\delta}^{* *}\right\|_{H^{-1}\left(0, T ; L^{2}\right)} \\
& =\sup _{\zeta \in H_{0}^{1}(0, T),\|\zeta\|_{H^{1}}=1}<\sup _{q \in L^{2},\|q\|_{L^{2}}=1}\left(p_{\delta}^{* *}(t), q\right), \zeta(t)>_{H^{-1}, H_{0}^{1}} \\
& \leq C \sup _{\zeta \in H_{0}^{1}(0, T),\|\zeta\|_{H^{1}}=1}<\sup _{\mathbf{v} \in \mathbf{H}_{c, 0},\|\mathbf{v}\|_{\mathbf{H}^{1}}=1}\left(p_{\delta}^{* *}(t), \nabla \cdot \mathbf{v}\right), \zeta(t)>_{H^{-1}, H_{0}^{1}} \\
& =C \sup _{\zeta \in H_{0}^{1}(0, T),\|\zeta\|_{H^{1}}=1}<\sup _{\mathbf{v} \in \mathbf{W},\|\underline{\mathbf{v}}\|_{\mathbf{W}}=1}-b\left(p_{\delta}^{* *}(t), \underline{\mathbf{v}}\right), \zeta(t)>_{H^{-1}, H_{0}^{1}} \\
& =C \sup _{\zeta \in H_{0}^{1}(0, T),\|\zeta\|_{H^{1}}=1}\left\langle\operatorname { s u p } _ { \underline { \mathbf { v } } \in \mathbf { W } , \| \mathbf { v } \| _ { \mathbf { W } } = 1 } \left(\left\langle\left(\begin{array}{c}
\frac{\mathrm{d} \mathbf{u}_{\delta}^{*}}{\mathrm{~d} t} \\
\eta \frac{\mathrm{d} \phi_{\delta}^{*}}{\mathrm{~d} t}
\end{array}\right), \underline{\mathbf{v}}\right\rangle\right.\right. \\
& \left.+a_{\eta}\left(\underline{\mathbf{u}}_{\delta}^{* *}, \underline{\mathbf{v}}\right)+\left\langle F^{m}, \underline{\mathbf{v}}>\right), \zeta(t)\right\rangle_{H^{-1}, H_{0}^{1}} \\
& \leq C \sup _{\zeta \in H_{0}^{1}(0, T),\|\zeta\|_{H^{1}}=1}\left(\left\langle\sup _{\underline{\mathbf{v}} \in \mathbf{W},\|\mathbf{v}\|_{\mathbf{W}}=1}-\left\langle\left(\begin{array}{c}
\mathbf{u}_{\delta}^{*} \\
\eta \phi_{\delta}^{*}
\end{array}\right), \underline{\mathbf{v}}\right\rangle, \zeta^{\prime}(t)\right\rangle\right. \\
& +\left\langle\sup _{\underline{\mathbf{v}} \in \mathbf{W},\|\mathbf{v}\|_{\mathbf{w}}=1} a_{\eta}\left(\underline{\mathbf{u}}_{\delta}^{* *}, \underline{\mathbf{v}}\right), \zeta(t)\right\rangle \\
& \left.+\left\langle\sup _{\underline{\mathbf{v}} \in \mathbf{W},\|\mathbf{v}\|_{\mathbf{W}}=1}\left\langle F^{m}, \underline{\mathbf{v}}\right\rangle, \zeta(t)\right\rangle\right) \\
& \leq C\left(\left\|\sup _{\underline{\mathbf{v}} \in \mathbf{W},\|\mathbf{v}\|_{\mathbf{w}}=1}\left\langle\left(\begin{array}{c}
\mathbf{u}_{\delta}^{*} \\
\eta \phi_{\delta}^{*}
\end{array}\right), \underline{\mathbf{v}}\right\rangle\right\|_{L^{2}(0, T)}\right. \\
& +\sup _{\underline{\mathbf{v}} \in \mathbf{W},\|\mathbf{v}\|_{\mathbf{w}}=1} a_{\eta}\left(\underline{\mathbf{u}}_{\delta}^{* *}, \underline{\mathbf{v}}\right) \|_{L^{2}(0, T)} \\
& \left.+\left\|\sup _{\underline{\mathbf{v}} \in \mathbf{W},\|\underline{\mathbf{v}}\|_{\mathbf{W}}=1}<F^{m}, \underline{\mathbf{v}}>\right\|_{L^{2}(0, T)}\right) \\
& \leq C\left(\left\|\underline{\mathbf{u}}_{\delta}^{*}\right\|_{L^{2}\left(0, T ; \mathbf{L}^{2} \times L^{2}\right)}+\left\|\underline{\mathbf{u}}_{\delta}^{* *}\right\|_{L^{2}(0, T ; \mathbf{W})}+\left\|F_{\delta}\right\|_{L^{2}\left(0, T ; \mathbf{W}^{\prime}\right)}\right) \leq C,
\end{aligned}
$$

where, in the second step, we have used the fact that the divergence operator is an isomorphism from $\mathbf{V}^{\perp}$ in $\mathbf{H}_{c, 0}$ to $L^{2}$, which is equivalent to the inf-sup condition proven in Lemma 3.1; see [22, Lem. 4.1, pp. 58]. Here, $\mathbf{V}^{\perp}$ denotes the orthogonal complement of $\mathbf{V}$ in $\mathbf{H}_{c, 0}$ with respect to the inner product $(\nabla \cdot, \nabla \cdot)$. The isomorphism gives that $\|\nabla \cdot \mathbf{v}\|_{\mathbf{L}^{2}} \geq \beta\|\mathbf{v}\|_{\mathbf{H}^{1}}$ for all $\mathbf{v} \in \mathbf{V}^{\perp}$. Thus, the ball $\left\{q:\|q\|_{L^{2}} \leq 1\right\}$ is a subset of $\left\{\nabla \cdot \mathbf{v}:\|\mathbf{v}\|_{\mathbf{H}^{1}} \leq 1 / \beta\right\}$.

The uniform bound on $\left\{p_{\delta}^{* *}\right\}$ in the Hilbert space $H^{-1}\left(0, T ; L^{2}\right)$ implies that we can extract a subsequence (without changing the notation) such that

$$
p_{\delta}^{* *} \underset{\delta \rightarrow 0}{\stackrel{w}{\longrightarrow}} p
$$

weakly in $H^{-1}\left(0, T ; L^{2}\right)$.

Next, we pass the limit in (4.4). For this purpose, let $\zeta \in C^{1}([0, T])$ with $\zeta(T)=0$ and $\underline{\mathbf{v}} \in \mathbf{W}$. We have

$$
\begin{aligned}
& \int_{0}^{T}\left(\left\langle-\left(\begin{array}{c}
\mathbf{u}_{\delta}^{*} \\
\eta \phi_{\delta}^{*}
\end{array}\right), \underline{\mathbf{v}} \zeta^{\prime}(t)\right\rangle+a_{\eta}\left(\underline{\mathbf{u}}_{\delta}^{* *}(t), \underline{\mathbf{v}}\right) \zeta(t)+b\left(\underline{\mathbf{v}} \zeta(t), p_{\delta}^{* *}\right)\right) \mathrm{d} t \\
= & \left\langle\left(\begin{array}{c}
\mathbf{u}_{0} \\
\eta \phi_{0}
\end{array}\right), \underline{\mathbf{v}}\right\rangle \zeta(0)+\int_{0}^{T}\left\langle F_{\delta}(t), \underline{\mathbf{v}}\right\rangle \zeta(t) \mathrm{d} t .
\end{aligned}
$$

Letting $\delta \rightarrow 0$ and utilizing the convergence of $\underline{\mathbf{u}}_{\delta}^{*}, \underline{\mathbf{u}}_{\delta}^{* *}, p_{\delta}^{* *}$, and $F_{\delta}$, we have

$$
\begin{aligned}
& \int_{0}^{T}\left(\left\langle-\left(\begin{array}{c}
\mathbf{u} \\
\eta \phi
\end{array}\right), \underline{\mathbf{v}} \zeta^{\prime}(t)\right\rangle+a_{\eta}(\underline{\mathbf{u}}, \underline{\mathbf{v}} \zeta(t))+b(\underline{\mathbf{v}} \zeta(t), p)\right) \mathrm{d} t \\
= & \left\langle\left(\begin{array}{c}
\mathbf{u}_{0} \\
\eta \phi_{0}
\end{array}\right), \underline{\mathbf{v}}\right\rangle \zeta(0)+\int_{0}^{T}\langle F(t), \underline{\mathbf{v}}\rangle \zeta(t) \mathrm{d} t
\end{aligned}
$$


which formally leads to the weak formulation (2.15) with the desired initial condition. In the case of $\mathbf{v} \in \mathbf{V}$, we recover the weak formulation (2.16). ${ }^{6}$

Thus, we have proven (2.16) and established the existence of the solution of $\underline{\mathbf{u}}$. Uniqueness of the solution (the velocity and hydraulic head) is straightforward due to the quasi-coercivity and the Gronwall inequality.

We may improve the weak convergence of the approximate solutions to strong convergence by invoking a compactness theorem due to Témam [24, Thm. 13.3] that states the following. Let $X$ and $Y$ denote two (not necessarily reflexive) $B a$ nach spaces with $Y \subset X$, the injection being compact. Suppose $\mathcal{G}$ is a set of functions in $L^{1}(\mathbb{R} ; Y) \cap L^{p}(\mathbb{R} ; X), p>1$, with $\mathcal{G}$ being bounded in $L^{p}(\mathbb{R} ; X)$ and $L^{1}(\mathbb{R} ; Y)$; $\int_{-\infty}^{+\infty}\|g(a+s)-g(s)\|_{X}^{p} d s \rightarrow 0$ as $a \rightarrow 0$ uniformly for $g \in \mathcal{G}$; and the support of the functions $g \in \mathcal{G}$ is included in a fixed compact set of $\mathbb{R}$, say $[-L,+L]$. Then, the set $\mathcal{G}$ is relatively compact in $L^{p}(\mathbb{R} ; X)$.

For the application we consider, we set $X=\mathbf{L}^{2} \times L^{2}, Y=\mathbf{V}$ and $p=2$. We take $\left\{\underline{\mathbf{u}}_{\delta}^{*}\right\}$ as $\mathcal{G}$. We extend $\left\{\underline{\mathbf{u}}_{\delta}^{*}\right\}$ by zero from the interval $[0, T]$ to the real line $\mathbb{R}$. Their boundedness in $L^{1}(\mathbb{R} ; \mathbf{V})$ and $L^{2}\left(\mathbb{R} ; \mathbf{L}^{2} \times L^{2}\right)$ is already shown by (4.5). It remains to show that

$$
\int_{-\infty}^{+\infty}\left\|\underline{\mathbf{u}}_{\delta}^{*}(a+s)-\underline{\mathbf{u}}_{\delta}^{*}(s)\right\|_{\mathbf{L}^{2} \times L^{2}}^{2} \mathrm{~d} s \rightarrow 0 \quad \text { as } a \rightarrow 0
$$

uniformly for all $\delta$. Without loss of generality, we assume $a>0$. Then,

$$
\begin{aligned}
& \int_{-\infty}^{+\infty}\left\|\underline{\mathbf{u}}_{\delta}^{*}(a+s)-\underline{\mathbf{u}}_{\delta}^{*}(s)\right\|_{\mathbf{L}^{2} \times L^{2}}^{2} \mathrm{~d} s \\
= & \int_{0}^{T-a}\left\|\underline{\mathbf{u}}_{\delta}^{*}(a+s)-\underline{\mathbf{u}}_{\delta}^{*}(s)\right\|_{\mathbf{L}^{2} \times L^{2}}^{2} \mathrm{~d} s+\int_{0}^{a}\left\|\underline{\mathbf{u}}_{\delta}^{*}(s)\right\|_{\mathbf{L}^{2} \times L^{2}}^{2} \mathrm{~d} s \\
& \quad+\int_{T-a}^{T}\left\|\underline{\mathbf{u}}_{\delta}^{*}(s)\right\|_{\mathbf{L}^{2} \times L^{2}}^{2} \mathrm{~d} s \\
\leq & 2 a\left\|\underline{\mathbf{u}}_{\delta}^{*}\right\|_{L^{\infty}\left(0, T ; \mathbf{L}^{2} \times L^{2}\right)}^{2}+\int_{0}^{T-a}\left\|\underline{\mathbf{u}}_{\delta}^{*}(a+s)-\underline{\mathbf{u}}_{\delta}^{*}(s)\right\|_{\mathbf{L}^{2} \times L^{2}}^{2} \mathrm{~d} s .
\end{aligned}
$$

We are thus done with the proof of the strong convergence of $\left\{\underline{\mathbf{u}}_{\delta}^{*}\right\}$ in $L^{2}\left(0, T ; \mathbf{L}^{2} \times L^{2}\right)$ if we can show that the last integral goes to 0 as $a \rightarrow 0$. For this purpose, we integrate (4.3) in time from $s$ to $a+s$ to yield

$$
\left\langle\left(\begin{array}{c}
\mathbf{u}_{\delta}^{*}(a+s)-\mathbf{u}_{\delta}^{*}(s) \\
\eta\left(\phi_{\delta}^{*}(a+s)-\phi_{\delta}^{*}(s)\right)
\end{array}\right), \underline{\mathbf{v}}\right\rangle=\int_{s}^{a+s}<F_{\delta}(t), \underline{\mathbf{v}}>-a_{\eta}\left(\underline{\mathbf{u}}_{\delta}^{* *}(t), \underline{\mathbf{v}}\right) \mathrm{d} t .
$$

Due to the continuity of $a_{\eta}(\cdot, \cdot)$ and the Hölder's inequality, we know

$$
\begin{aligned}
& \int_{s}^{a+s}\left|a_{\eta}\left(\underline{\mathbf{u}}_{\delta}^{* *}(t), \underline{\mathbf{v}}\right)\right| \mathrm{d} t \leq C_{\eta} \int_{s}^{a+s}\left\|\underline{\mathbf{u}}_{\delta}^{* *}(t)\right\|_{\mathbf{V}}\left\|_{\mathbf{v}}\right\|_{\mathbf{V}} \mathrm{d} t \\
\leq & C_{\eta}\left(\int_{s}^{a+s}\left\|\underline{\mathbf{u}}_{\delta}^{* *}(t)\right\|_{\mathbf{V}}^{2} \mathrm{~d} t\right)^{1 / 2}\left(\int_{s}^{a+s}\|\underline{\mathbf{v}}\|_{\mathbf{V}}^{2} \mathrm{~d} t\right)^{1 / 2} \\
\leq & C_{\eta} a^{1 / 2}\|\underline{\mathbf{v}}\|_{\mathbf{V}}\left\|\underline{\mathbf{u}}_{\delta}^{* *}\right\|_{L^{2}(0, T ; \mathbf{V})} .
\end{aligned}
$$

\footnotetext{
${ }^{6}$ To show that the above duality is equivalent to (2.16) with the proper initial condition, we actually need to justify the integration by parts (or the Green's formula) we have used and to show the continuity of the solution $\underline{\mathbf{u}}$ with value in $\mathbf{L}^{2} \times L^{2}$. This requires the estimation of $\left\|\underline{\mathbf{u}}_{t}\right\|_{L^{2}\left(0, T ; \mathbf{V}^{\prime}\right)}$ which is derived earlier; see $[23,15]$ for a very similar context.
} 
Likewise, we have

$$
\int_{s}^{a+s}\left|<F_{\delta}(t), \underline{\mathbf{v}}>\right| \mathrm{d} t \leq C a^{1 / 2}\|\underline{\mathbf{v}}\|_{\mathbf{V}}\left\|F_{\delta}\right\|_{L^{2}\left(0, T ; \mathbf{V}^{\prime}\right)} .
$$

Now we can set $\underline{\mathbf{v}}=\underline{\mathbf{u}}_{\delta}^{*}(a+s)-\underline{\mathbf{u}}_{\delta}^{*}(s)$ in the time integration of (4.3), to deduce that ${ }^{7}$

$$
\begin{aligned}
& \int_{0}^{T-a}\left\|\underline{\mathbf{u}}_{\delta}^{*}(a+s)-\underline{\mathbf{u}}_{\delta}^{*}(s)\right\|_{\mathbf{L}^{2} \times L^{2}}^{2} \mathrm{~d} s \\
\leq & C_{\eta} a^{1 / 2}\left(\left\|\underline{\mathbf{u}}_{\delta}^{* *}\right\|_{L^{2}(0, T ; \mathbf{V})}+\left\|F_{\delta}\right\|_{L^{2}\left(0, T ; \mathbf{V}^{\prime}\right)}\right) \int_{0}^{T-a}\left\|\underline{\mathbf{u}}_{\delta}^{*}(a+s)-\underline{\mathbf{u}}_{\delta}^{*}(s)\right\|_{\mathbf{V}} \mathrm{d} s \\
\leq & C_{\eta} a^{1 / 2}\left\|\underline{\mathbf{u}}_{\delta}^{*}\right\|_{L^{1}(0, T ; \mathbf{V})} \leq C_{\eta} a^{1 / 2}\left\|\underline{\mathbf{u}}_{\delta}^{*}\right\|_{L^{2}(0, T ; \mathbf{V})} \leq C_{\eta} a^{1 / 2} .
\end{aligned}
$$

For the strong convergence of $\underline{\mathbf{u}}_{\delta}^{* *}$ to $\mathbf{u}$, we look at the difference between $\underline{\mathbf{u}}_{\delta}^{*}$ and $\underline{\mathbf{u}}_{\delta}^{* *}:$

$$
\begin{aligned}
& \left\|\underline{\mathbf{u}}_{\delta}^{*}-\underline{\mathbf{u}}_{\delta}^{* *}\right\|_{L^{2}\left(0, T ; \mathbf{L}^{2}\left(\Omega_{c}\right) \times L^{2}\left(\Omega_{m}\right)\right)}^{2}=\sum_{m=0}^{N-1} \int_{m \delta}^{(m+1) \delta}\left\|\underline{\mathbf{u}}_{\delta}^{*}-\underline{\mathbf{u}}_{\delta}^{* *}\right\|_{\mathbf{L}^{2} \times L^{2}}^{2} \mathrm{~d} t \\
= & \sum_{m=0}^{N-1} \int_{0}^{\delta}\left\|\frac{\underline{\mathbf{u}}_{\delta}^{m+1}-\underline{\mathbf{u}}_{\delta}^{m}}{\delta}\right\|_{\mathbf{L}^{2} \times L^{2}}^{2} t^{2} \mathrm{~d} t \\
= & \sum_{m=0}^{N-1}\left\|\underline{\mathbf{u}}_{\delta}^{m+1}-\underline{\mathbf{u}}_{\delta}^{m}\right\|_{\mathbf{L}^{2} \times L^{2}}^{2} \int_{0}^{\delta}\left(\frac{t}{\delta}\right)^{2} \mathrm{~d} t \leq C \delta .
\end{aligned}
$$

strongly in $L^{2}\left(0, T ; \mathbf{L}^{2}\left(\Omega_{c}\right) \times L^{2}\left(\Omega_{m}\right)\right)$. To summarize, we have the following result.

THEOREM 4.1. The weak formulation of the coupled Stokes-Darcy system with Beavers-Joseph interfacial boundary condition (2.16) is well-posed under the assumption of isotropic (but not necessarily homogeneous) hydraulic conductivity. Moreover, the solution to the backward Euler scheme (4.1) converges to the solution of the continuous system (2.16) as the time step $\delta$ approaches zero, i.e., $\underline{\mathbf{u}}_{\delta}^{*}$ and $\underline{\mathbf{u}}_{\delta}^{* *}$ converge to $\underline{\mathbf{u}}$ in $L^{2}\left(0, T ; \mathbf{L}^{2}\left(\Omega_{c}\right) \times L^{2}\left(\Omega_{m}\right)\right)$ as the time step $\delta$ approaches zero.

Proof. We have already shown the existence and the convergence of the numerical solution to a solution to the continuous-in-time system. We only need to show the continuous dependence on the initial data and forcing term $F$.

Let $\underline{\mathbf{u}}=\underline{\mathbf{u}}_{1}-\underline{\mathbf{u}}_{2}$, where $\underline{\mathbf{u}}_{1}$ and $\underline{\mathbf{u}}_{2}$ are two solutions to the weak formulation (2.16) with initial data $\underline{\mathbf{u}}_{01}$ and $\underline{\mathbf{u}}_{02}$ and forcing term $\widetilde{F}_{1}$ and $\widetilde{F}_{2}$, respectively. Then, $\underline{\mathbf{u}}$ satisfies (2.16) with initial data $\underline{\mathbf{u}}_{0}=\underline{\mathbf{u}}_{01}-\underline{\mathbf{u}}_{02}$ and forcing term $\widetilde{F}=\widetilde{F}_{1}-\widetilde{F}_{2}$. Formally setting $\underline{\mathbf{v}}$ to $\underline{\mathbf{u}}$ in (2.16) and utilizing the Gårding type estimate (4.2) we have

$$
\frac{1}{2} \frac{d\|\mathbf{u}\|_{\mathbf{L}^{2}}^{2}}{d t}+\frac{\eta}{2} \frac{d\|\phi\|_{L^{2}}^{2}}{d t}+\alpha_{\eta}\|\underline{\mathbf{u}}\|_{\mathbf{V}}^{2}-C_{0}\|\underline{\mathbf{u}}\|_{\mathbf{L}^{2} \times L^{2}}^{2} \leq\|F\|_{\mathbf{V}^{\prime}}\|\underline{\mathbf{u}}\|_{\mathbf{V}}
$$

which leads to continuous dependence of the solution (in particular uniqueness) on the initial data and external forcing term after we apply the Cauchy-Schwarz inequality, the Poincaré inequality, and the Gronwall inequality.

The semi-discrete scheme that we used in our existence analysis can be further discretized in space if we are interested in a fully discrete numerical scheme. Indeed,

\footnotetext{
${ }^{7}$ The $C$ 's and $C_{\eta}$ 's may denote different constants from inequality to inequality.
} 
at each time step we also know the convergence of spatially discretized solution of (4.1) according to the finite element analysis conducted in section 3. Although the stationary problem and the backward Euler discretization are slightly different, the same analysis given in that section carries over if we just take $a_{\delta, \eta}(\cdot, \cdot)$ as $a(\cdot, \cdot)$. Then, we have solutions to the spatially and temporally discretized problem. They actually converge to the solution of the continuous problem as $h$ and $\delta$ are reduced. To show this, for a fixed $N$, we denote the piecewise constant interpolation of solutions of the fully discretized problem by $\underline{\mathbf{u}}_{h, \delta}^{* *}$, i.e.,

$$
\underline{\mathbf{u}}_{h, \delta}^{* *}(t)=\underline{\mathbf{u}}_{h, \delta}^{m+1} \quad \text { for } t \in(m \delta,(m+1) \delta],
$$

where $\underline{\mathbf{u}}_{h, \delta}^{m+1}$ is the solution of the following fully discretized problem with mesh size $h$,

$$
\begin{array}{rlrl}
\frac{1}{\delta}\left\langle\left(\begin{array}{c}
\mathbf{u}_{h, m}^{m+1}-\mathbf{u}_{h, \delta}^{m} \\
\eta\left(\phi_{h, \delta}^{m+1}-\phi_{h, \delta}^{m}\right)
\end{array}\right), \underline{\mathbf{v}}_{h}\right\rangle & +a_{\eta}\left(\underline{\mathbf{u}}_{h, \delta}^{m+1}, \underline{\mathbf{v}}_{h}\right) & \\
+ & b\left(\underline{\mathbf{v}}_{h}, p_{h, \delta}^{m+1}\right)=<F^{m}, \underline{\mathbf{v}}_{h}> & & \forall \underline{\mathbf{v}}_{h} \in \mathbf{W}^{h} \\
b\left(\underline{\mathbf{u}}_{h, \delta}^{m+1}, q^{h}\right) & =0 & & \forall q \in Q^{h} .
\end{array}
$$

We know that $\underline{\mathbf{u}}_{h, \delta}^{m+1} \rightarrow \underline{\hat{\mathbf{u}}}_{\delta}^{m+1}$ strongly in $\mathbf{W}$ as $h \rightarrow 0$, where $\underline{\hat{\mathbf{u}}}_{\delta}^{m+1}$ is the exact solution of the above problem for a given $\underline{\mathbf{u}}_{h, \delta}^{m}$. We denote $\left\|\underline{\mathbf{u}}_{h, \delta}^{m+1}-\underline{\hat{\mathbf{u}}}_{\delta}^{m+1}\right\|_{\mathbf{W}}$ by $\tau^{m+1}$ and $\left\|\underline{\mathbf{u}}_{h, \delta}^{m+1}-\underline{\mathbf{u}}_{\delta}^{m+1}\right\|_{\mathbf{w}}$ by $\varepsilon^{m+1}$. Then, by the stability of problem (4.1), we know that

$$
\begin{aligned}
& \varepsilon^{m+1}=\left\|\underline{\mathbf{u}}_{h, \delta}^{m+1}-\underline{\mathbf{u}}_{\delta}^{m+1}\right\|_{\mathbf{w}} \\
\leq & \left\|\underline{\mathbf{u}}_{h, \delta}^{m+1}-\underline{\hat{\mathbf{u}}}_{\delta}^{m+1}\right\| \mathbf{W}+\left\|\underline{\hat{\mathbf{u}}}_{\delta}^{m+1}-\underline{\mathbf{u}}_{\delta}^{m+1}\right\| \mathbf{w} \leq \tau^{m+1}+C \varepsilon^{m+1},
\end{aligned}
$$

where $C$ is independent of $m$. Then, the error between the fully discretized approximate solution and the temporally discretized approximate solution is given by

$$
\left\|\underline{\mathbf{u}}_{h, \delta}^{* *}-\underline{\mathbf{u}}_{\delta}^{* *}\right\|_{L^{2}(0, T ; \mathbf{W})}^{2}=\sum_{m=0}^{N-1} \int_{0}^{\delta}\left(\varepsilon^{m+1}\right)^{2} \mathrm{~d} t \leq T\left(\sup _{m} \varepsilon^{m+1}\right)^{2} .
$$

Now, for a fixed $N$, we simply denote $\sup _{m} \tau^{m+1}$ by $\tau$. By induction on (4.7), the error at time $(m+1) \delta$ will have the following upper bound:

$$
\varepsilon^{m+1} \leq \tau \sum_{i=0}^{m} C^{i} \quad \forall m
$$

Then, $\sup _{m} \varepsilon^{m+1} \leq \tau \sum_{i=0}^{N-1} C^{i} \rightarrow 0$ as $\tau \rightarrow 0$, i.e., $\left\|\underline{\mathbf{u}}_{h, \delta}^{* *}-\underline{\mathbf{u}}_{\delta}^{* *}\right\|_{L^{2}(0, T ; \mathbf{W})}^{2} \rightarrow 0$ as $h \rightarrow 0$ by estimate (4.8). To summarize, we have the following result.

THEOREM 4.2. The fully discretized solutions $\underline{\mathbf{u}}_{h, \delta}^{* *}$ converge to $\underline{\mathbf{u}}$ weakly in $L^{2}(0, T ; \mathbf{W})$ and strongly in $L^{2}\left(0, T ; \mathbf{L}^{2} \times L^{2}\right)$ as $h \rightarrow 0$ and $\delta \rightarrow 0$, with the limits taken in that order. More precisely, we have

$$
\lim _{\delta \rightarrow 0} \lim _{h \rightarrow 0} \underline{\mathbf{u}}_{h, \delta}^{* *}=\underline{\mathbf{u}} .
$$


Similarly, we have the weak convergence of the pressure in $H^{-1}\left(0, T ; L^{2}\right)$. The convergence we have shown here is not associated with any rate. In [12], the convergence rates of finite element approximations to the time-dependent Stokes-Darcy problem are discussed.

We also point out that in the case for which the bilinear term $a_{\eta}$ is coercive (such as is the case for sufficiently small $\alpha$ in the Beavers-Joseph condition; see section 3 ) and the external forcing term is time-independent, all time-dependent solutions converge to the unique time-independent solution as time goes to infinity.

Appendix A. Equivalence. We briefly show the equivalence between the solution to the classical formulation $(2.2)-(2.8)$ and the solution to weak formulation (2.14), provided that the latter is sufficiently smooth. In the following argument, we follow the notational convention introduced earlier and assume that $\underline{\mathbf{u}}=(\mathbf{u}, \phi)$, where $\mathbf{u} \in \mathbf{H}_{c, f}, \underline{\mathbf{v}}=(\mathbf{v}, \psi) \in \mathbf{W}$, and $p \in Q$.

First, we investigate the time-dependent Navier-Stokes equations with gravitational forcing:

$$
\mathbf{u}_{t}+(\mathbf{u} \cdot \nabla) \mathbf{u}=\nabla(-p \mathbb{I}+2 \nu \mathbb{D}(\mathbf{u}))-g \mathbf{k} .
$$

This implies that

$$
\int_{\Omega_{c}}\left(\mathbf{u}_{t}+(\mathbf{u} \cdot \nabla) \mathbf{u}\right) \cdot \mathbf{v} \mathrm{d} \Omega_{c}=\int_{\Omega_{c}}(\nabla(-p \mathbb{I}+2 \nu \mathbb{D}(\mathbf{u}))-g \mathbf{k}) \cdot \mathbf{v} \mathrm{d} \Omega_{c}
$$

for all $\underline{\mathbf{v}}=(\mathbf{v}, \psi) \in \mathbf{W}$. Integrating by parts, we have:

$$
\begin{aligned}
\text { R.H.S. }= & \int_{\partial \Omega_{c}}[(-p \mathbb{I}+2 \nu \mathbb{D}(\mathbf{u})) \mathbf{n}] \cdot \mathbf{v} \mathrm{d} \Gamma_{c} \\
& -\int_{\Omega_{c}}(-p \mathbb{I}+2 \nu \mathbb{D}(\mathbf{u})): \nabla \mathbf{v} \mathrm{d} \Omega_{c}-\int_{\Omega_{c}} g \mathbf{k} \cdot \mathbf{v} \mathrm{d} \Omega_{c} \\
= & \int_{\Gamma_{c m}}[(-p \mathbb{I}+2 \nu \mathbb{D}(\mathbf{u})) \mathbf{n}] \cdot \mathbf{v} \mathrm{d} \Gamma_{c m}-\int_{\Omega_{c}}(-p \mathbf{I}+2 \nu \mathbb{D}(\mathbf{u})): \nabla \mathbf{v} \mathrm{d} \Omega_{c} \\
& \quad-\int_{\Omega_{c}} g \mathbf{k} \cdot \mathbf{v} \mathrm{d} \Omega_{c} \\
= & \int_{\Omega_{c}} p \nabla \cdot \mathbf{v} \mathrm{d} \Omega_{c}-\int_{\Omega_{c}} 2 \nu \mathbb{D}(\mathbf{u}): \mathbb{D}(\mathbf{v}) \mathrm{d} \Omega_{c}-\int_{\Omega_{c}} g \mathbf{k} \cdot \mathbf{v} \mathrm{d} \Omega_{c} \\
& +\int_{\Gamma_{c m}}\left(\mathbf{n}^{T}(-p \mathbb{I}+2 \nu \mathbb{D}(\mathbf{u})) \mathbf{n}\right) \mathbf{v} \cdot \mathbf{n} \mathrm{d} \Gamma_{c m} \\
& +\int_{\Gamma_{c m}} P_{\tau}((-p \mathbb{I}+2 \nu \mathbb{D}(\mathbf{u})) \mathbf{n}) \cdot P_{\tau}(\mathbf{v}) \mathrm{d} \Gamma_{c m} \\
= & \int_{\Omega_{c}} p \nabla \cdot \mathbf{v} \mathrm{d} \Omega_{c}-\int_{\Omega_{c}} 2 \nu \mathbb{D}(\mathbf{u}): \mathbb{D}(\mathbf{v}) \mathrm{d} \Omega_{c}-\int_{\Omega_{c}} g \mathbf{k} \cdot \mathbf{v} \mathrm{d} \Omega_{c} \\
& +\int_{\Gamma_{c m}}\left(\mathbf{n}^{T}(-p \mathbb{I}+2 \nu \mathbb{D}(\mathbf{u})) \mathbf{n}\right) \mathbf{v} \cdot \mathbf{n} \mathrm{d} \Gamma_{c m} \\
& +\int_{\Gamma_{c m}} P_{\tau}((-p \mathbb{I}+2 \nu \mathbb{D}(\mathbf{u})) \mathbf{n}) \cdot \mathbf{v} \mathrm{d} \Gamma_{c m} .
\end{aligned}
$$


Substituting this into the interface condition, we arrive at

$$
\begin{aligned}
\int_{\Omega_{c}}\left(\mathbf{u}_{t}+(\mathbf{u} \cdot \nabla) \mathbf{u}\right) \cdot \mathbf{v} \mathrm{d} \Omega_{c}= & \int_{\Omega_{c}} p \nabla \cdot \mathbf{v} \mathrm{d} \Omega_{c}-\int_{\Omega_{c}} 2 \nu \mathbb{D}(\mathbf{u}): \mathbb{D}(\mathbf{v}) \mathrm{d} \Omega_{c} \\
& -\int_{\Omega_{c}} g \mathbf{k} \cdot \mathbf{v} \mathrm{d} \Omega_{c}-\int_{\Gamma_{c m}} g(\phi-z) \mathbf{v} \cdot \mathbf{n} \mathrm{d} \Gamma_{c m} \\
& -\int_{\Gamma_{c m}} \frac{\nu \alpha \sqrt{3}}{\sqrt{\operatorname{trace}(\boldsymbol{\Pi})}} P_{\tau}(\mathbf{u}+\mathbb{K} \nabla \phi) \cdot \mathbf{v} \mathrm{d} \Gamma_{c m} .
\end{aligned}
$$

Next, we write down the variational form for the Darcy equation (divided by $S$ and multiplied by the rescaling parameter $\eta$ ):

$$
\begin{aligned}
& \frac{\eta}{S} \int_{\Omega_{m}}\left(\phi_{t}+\nabla \cdot(-\mathbb{K} \nabla \phi)\right) \psi \mathrm{d} \Omega_{m} \\
= & \int_{\Omega_{m}} \eta \phi_{t} \psi \mathrm{d} \Omega_{m}+\frac{\eta}{S} \int_{\partial \Omega_{m}}(-\mathbb{K} \nabla \phi) \cdot \mathbf{n} \psi \mathrm{d} \Gamma_{m}+\frac{\eta}{S} \int_{\Omega_{m}}(\mathbb{K} \nabla \phi) \cdot \nabla \psi \mathrm{d} \Omega_{m} \\
= & \int_{\Omega_{m}} \eta \phi_{t} \psi \mathrm{d} \Omega_{m}+\frac{\eta}{S} \int_{\Gamma_{c m}}(-\mathbb{K} \nabla \phi) \cdot \mathbf{n} \psi \mathrm{d} \Gamma_{c m}+\frac{\eta}{S} \int_{\Omega_{m}}(\mathbb{K} \nabla \phi) \cdot \nabla \psi \mathrm{d} \Omega_{m} \\
= & \int_{\Omega_{m}} \eta \phi_{t} \psi \mathrm{d} \Omega_{m}-\frac{\eta}{S} \int_{\Gamma_{c m}} \mathbf{u} \cdot \mathbf{n}_{c m} \psi \mathrm{d} \Gamma_{c m}+\frac{\eta}{S} \int_{\Omega_{m}}(\mathbb{K} \nabla \phi) \cdot \nabla \psi \mathrm{d} \Omega_{m},
\end{aligned}
$$

where $\mathbf{n}=-\mathbf{n}_{c m}$.

Now, summing up the above variational forms, dropping the non-linear term, using the bilinear forms, linear functional, and the dual defined in section 2, and including the div-free condition, we arrive at the weak formulation (2.14), i.e.,

$$
\left\{\begin{aligned}
\left\langle\underline{\mathbf{u}}_{t}, \underline{\mathbf{v}}\right\rangle_{\eta}+a_{\eta}(\underline{\mathbf{u}}, \underline{\mathbf{v}})+b(\underline{\mathbf{v}}, p) & =\langle F, \underline{\mathbf{v}}\rangle \\
b(\underline{\mathbf{u}}, q) & =0
\end{aligned}\right.
$$

Thus, we have shown that a solution to problem (2.2)-(2.8) is a solution to (2.14). Next, we want to show that the solution to the weak formulation defined above is a solution to problem (2.2)-(2.8), provided the weak solution is smooth enough. In fact, we just need to reverse the above argument. Following the classical argument, by setting $\mathbf{v}=\mathbf{0}$ in the test function and letting $\psi \in H_{p}$ be arbitrary, we can show that the equality (2.2) and the first condition in interface condition (2.8) hold in the proper sense. By setting $\psi=0$ in the test function $\underline{\mathbf{v}}$ and letting $\mathbf{v} \in \mathbf{H}_{c, 0}$ be arbitrary, we have that equality (2.5) and the the other two conditions in (2.8) hold in the proper sense as well.

Appendix B. Finite element approximations. In this subsection, we continue the discussion of section 3 and give an error bound for finite element approximations. Following the notations and spaces defined in section 3, we know that the div-stability condition guarantees that $\mathbf{V}_{f}^{h}$ is not empty. We choose such a $\underline{\mathbf{u}}_{0}^{h}$ in $\mathbf{V}_{f}^{h}$ and solve the problem: find $\underline{\mathbf{z}}^{h}$ in $\mathbf{V}^{h}$ such that

$$
a\left(\underline{\mathbf{z}}^{h}, \underline{\mathbf{v}}^{h}\right)=\left\langle l, \underline{\mathbf{v}}^{h}\right\rangle-a\left(\underline{\mathbf{u}}_{0}^{h}, \underline{\mathbf{v}}^{h}\right) \quad \forall \underline{\mathbf{v}}^{h} \in \mathbf{V}^{h} .
$$

By the Lax-Milgram theorem, this problem has a unique solution $\underline{\mathbf{z}}^{h}$; then, $\underline{\mathbf{u}}^{h}:=$ $\underline{\mathbf{u}}_{0}^{h}+\underline{\mathbf{z}}^{h}$, is the solution to the discrete problem. 
Now, for all $\underline{\mathbf{w}}^{h} \in \mathbf{V}_{f}^{h}$, let $\underline{\mathbf{v}}^{h}:=\underline{\mathbf{u}}^{h}-\underline{\mathbf{w}}^{h} \in \mathbf{V}^{h} ;$ then $^{8}$

$$
\begin{aligned}
& a\left(\underline{\mathbf{v}}^{h}, \underline{\mathbf{v}}^{h}\right)=\left\langle l, \underline{\mathbf{v}}^{h}\right\rangle-a\left(\underline{\mathbf{w}}^{h}, \underline{\mathbf{v}}^{h}\right)=a\left(\underline{\mathbf{u}}, \underline{\mathbf{v}}^{h}\right)+b\left(\underline{\mathbf{v}}^{h}, p\right)-a\left(\underline{\mathbf{w}}^{h}, \underline{\mathbf{v}}^{h}\right) \\
= & a\left(\underline{\mathbf{u}}-\underline{\mathbf{w}}^{h}, \mathbf{v}^{h}\right)+b\left(\mathbf{v}^{h}, p-q^{h}\right) \\
\leq & \|a\|\left\|\underline{\mathbf{u}}-\underline{\mathbf{w}}^{h}\right\|_{\mathbf{W}}\left\|\underline{\mathbf{v}}^{h}\right\|_{\mathbf{w}}+\|b\|\left\|\underline{\mathbf{v}}^{h}\right\|_{\mathbf{w}}\left\|p-q^{h}\right\|_{L^{2}} \quad \forall q^{h} \in Q^{h} .
\end{aligned}
$$

By coercivity, we have

$$
\left\|\underline{\mathbf{v}}^{h}\right\|_{\mathbf{W}} \leq \frac{1}{\alpha}\left(\|a\|\left\|\underline{\mathbf{u}}-\underline{\mathbf{w}}^{h}\right\|_{\mathbf{W}}+\|b\|\left\|p-q^{h}\right\|_{L^{2}}\right) .
$$

Therefore, since $\left\|\underline{\mathbf{u}}-\underline{\mathbf{u}}^{h}\right\| \leq\left\|\underline{\mathbf{u}}-\underline{\mathbf{w}}^{h}\right\|+\left\|\underline{\mathbf{w}}^{h}-\underline{\mathbf{u}}^{h}\right\|$,

$$
\left\|\underline{\mathbf{u}}-\underline{\mathbf{u}}^{h}\right\|_{\mathbf{W}} \leq \frac{1}{\alpha}\left((1+\|a\|)\left\|\underline{\mathbf{u}}-\underline{\mathbf{w}}^{h}\right\|_{\mathbf{W}}+\|b\|\left\|p-q^{h}\right\|_{L^{2}}\right) \quad \forall \underline{\mathbf{w}}^{h} \in \mathbf{V}_{f}^{h}, q^{h} \in Q^{h} .
$$

Furthermore, div-stability gives the following bound:

$$
\inf _{\underline{\mathbf{w}}^{h} \in \mathbf{V}_{f}^{h}}\left\|\underline{\mathbf{u}}-\underline{\mathbf{w}}^{h}\right\| \mathbf{w} \leq\left(1+\frac{\|b\|}{\beta}\right) \inf _{\underline{\mathbf{v}}^{h} \in \mathbf{W}^{h}}\left\|\underline{\mathbf{u}}-\underline{\mathbf{v}}^{h}\right\| \mathbf{w} .
$$

Thus, we arrive at the following estimate:

$$
\left\|\underline{\mathbf{u}}-\underline{\mathbf{u}}^{h}\right\|_{\mathbf{W}}<C\left(\inf _{\underline{\mathbf{v}}^{h} \in \mathbf{W}^{h}}\left\|\underline{\mathbf{u}}-\underline{\mathbf{v}}^{h}\right\|_{\mathbf{W}}+\inf _{q^{h} \in Q^{h}}\left\|p-q^{h}\right\|_{L^{2}}\right) .
$$

It remains to estimate $\left\|p-p^{h}\right\|_{L^{2}}$. First, we have

$$
\begin{aligned}
b\left(\underline{\mathbf{v}}^{h}, p^{h}-q^{h}\right) & =b\left(\underline{\mathbf{v}}^{h}, p^{h}\right)-b\left(\underline{\mathbf{v}}^{h}, q^{h}\right)=<l, \underline{\mathbf{v}}^{h}>-a\left(\underline{\mathbf{u}}^{h}, \underline{\mathbf{v}}^{h}\right)-b\left(\underline{\mathbf{v}}^{h}, q^{h}\right) \\
& =a\left(\underline{\mathbf{u}}, \underline{\mathbf{v}}^{h}\right)+b\left(\underline{\mathbf{v}}^{h}, p\right)-a\left(\underline{\mathbf{u}}^{h}, \underline{\mathbf{v}}^{h}\right)-b\left(\underline{\mathbf{v}}^{h}, q^{h}\right) \\
& =a\left(\underline{\mathbf{u}}-\underline{\mathbf{u}}^{h}, \underline{\mathbf{v}}^{h}\right)+b\left(\underline{\mathbf{v}}^{h}, p-q^{h}\right) \quad \forall \underline{\mathbf{v}}^{h} \in \mathbf{W}^{h}, q^{h} \in Q^{h} .
\end{aligned}
$$

Then, by div-stability, we have

$$
\begin{aligned}
\left\|p^{h}-q^{h}\right\|_{L^{2}} & \leq \frac{1}{\beta} \sup _{\underline{\mathbf{v}}^{h} \in \mathbf{W}} \frac{1}{\left\|\underline{\mathbf{v}}^{h}\right\|_{\mathbf{W}}}\left(a\left(\underline{\mathbf{u}}-\underline{\mathbf{u}}^{h}, \underline{\mathbf{v}}^{h}\right)+b\left(\underline{\mathbf{v}}^{h}, p-q^{h}\right)\right) \\
& \leq \frac{1}{\beta}\left(\|a\|\left\|\underline{\mathbf{u}}-\underline{\mathbf{u}}^{h}\right\|_{\mathbf{W}}+\|b\|\left\|p-q^{h}\right\|_{L^{2}}\right) \quad \forall q^{h} \in Q^{h} .
\end{aligned}
$$

Thus, by the triangle inequality $\left\|p-p^{h}\right\|_{L^{2}} \leq\left\|p-q^{h}\right\|_{L^{2}}+\left\|p^{h}-q^{h}\right\|_{L^{2}}$, we have

$$
\left\|p-p^{h}\right\|_{L^{2}} \leq \frac{1}{\beta}\left(\|a\|\left\|\underline{\mathbf{u}}-\underline{\mathbf{u}}^{h}\right\|_{\mathbf{W}}+(\beta+\|b\|) \inf _{q^{h} \in Q^{h}}\left\|p-q^{h}\right\|_{L^{2}}\right) .
$$

Acknowledgement. This work is supported in part by a grant from the National Science Foundation under grant numbers CMG DMS-0620035 (for MG, FH, and XW) and DMS-0620091 (for YC). This is also part of the dissertation of Fei Hua under the direction of Max Gunzburger and Xiaoming Wang. The financial support (for XW) from the Chinese MOE through an 111 project at Fudan University is also gratefully acknowledged. We also thank Bill Hu, Bill Layton, Jill Reese and Ming Ye for helpful conversation.

\footnotetext{
${ }^{8}\|a\|$ and $\|b\|$ here denote the norm of the bilinear terms $a(\cdot, \cdot)$ and $b(\cdot, \cdot)$.
} 


\section{REFERENCES}

[1] A. Quarteroni and A. Valli, Domain Decomposition Methods for Partial Differential Equations, Oxford Science Publications, Oxford, 1999.

[2] M. Discacciati and A. Quarteroni, Analysis of a domain decomposition method for the coupling of Stokes and Darcy equations, F. Brezzi, A. Buffa, S. Corsaro, A. Murli (eds), 2rd European Conference on Numerical Mathematics and Advanced Applications (ENUMATH 2001), Milan, Springer, 3-20, 2003.

[3] M. Discacciati, E. Miglio and A. Quarteroni, Mathematical and numerical models for coupling surface and groundwater flows, Appl. Numer. Math., 43, 57-74, 2002.

[4] M. Discacciati, A. Quarteroni and A. Valli, Robin-Robin domain decomposition methods for the Stokes-Darcy coupling, SIAM J. Numer. Anal., 45, 1246-1268, 2007.

[5] R. Glowinski, T. Pan and J. Periaux, A Lagrange multiplier/fictitious domain method for the numerical simulation of incompressible viscous flow around moving grid bodies: I. case where the rigid body motions are known a priori, C.R. Acad. Sci. Paris Sér. I Math., 324, 361-369, 1997.

[6] W.J. Layton, F. Schieweck and I. Yotov, Coupling fluid flow with porous media flow, SIAM J. Numer. Anal., 40, 2195-2218, 2003.

[7] M. Mu and J. Xu, A two-grid method of a mixed Stokes-Darcy model for coupling fluid flow with porous media flow, SIAM J. Numer. Anal., 45, 1801-1813, 2007.

[8] G. Beavers and D. Joseph, Boundary conditions at a naturally permeable wall, J. Fluid Mech., 30, 197-207, 1967.

[9] P. Saffman, On the boundary condition at the interface of a porous medium, Stud. in Appl. Math., 1, 77-84, 1971.

[10] I. P. Jones, Reynolds number flow past a porous spherical shell, Proc. Camb. Phil. Soc., 73, 231-238, 1973.

[11] W. JÄger and A. Mikelić, On the interface boundary condition of Beavers, Joseph and Saffman, SIAM J. Appl. Math., 60, 1111-1127, 2000.

[12] Y. Cao, M. Gunzburger, X. Hu, F. Hua, X. Wang and W. Zhao, Finite element approximation for time-dependent Stokes-Darcy flow with Beavers-Joseph interface boundary condition, SIAM J. Numer. Anal., submitted, 2008.

[13] J. Bear, Dynamics of Fluids in Porous Media, Dover, 1972

[14] J.L. Lions and E. Magenes, Problèmes Aux Limites Nonhomogènes et Applications, Dunod, Paris, 1, 1968.

[15] R. Temam, Navier-Stokes Equations: Theory and Numerical Analysis, AMS Chelsea Publishing, 2001.

[16] M. LeBars and M.G. Worster, Interfacial conditions between a pure fluid and a porous medium: implications for binary alloy solidification, J. Fluid Mech., 550, 149-173, 2006.

[17] X. Wang, Vanishing Darcy number limit of the Stokes-Brinkman model, in preparation, 2008.

[18] F. Brezzi and M. Fortin, Mixed and Hybrid Finite Element Methods, Springer-Verlag, New York, 1991.

[19] L.E. Payne and B. Straughan, Analysis of the boundary condition at the interface between a viscous fluid and a porous medium and related modelling questions, 77, 1998.

[20] V. John and J. Layton, Analysis of numerical errors in large eddy simulation, SIAM J. Numer. Anal., 40, 995-1020, 2002.

[21] M. Discacciati, Domain decomposition methods for the coupling of surface and groundwater flows, PhD thesis, École Polytechnique Fédérale de Lausanne, 2004.

[22] V. Girault and P.A. Raviart, Finite Element Methods for Navier-Stokes Equations: Theory and Algorithms, Springer-Verlag, 1986.

[23] V. Girault and P.A. Raviart, Finite Element Approximation of the Navier-Stokes Equations, (Lecture Notes in Mathematics), Springer-Verlag, Berlin, 1979.

[24] R. Temam, Navier-Stokes Equations and Nonlinear Functional Analysis, SIAM, 1995. 